ARTICLE

https://doi.org/10.1038/s41467-020-18565-8

\title{
CD36 facilitates fatty acid uptake by dynamic palmitoylation-regulated endocytosis
}

Jian-Wei Hao ${ }^{1,6}$, Juan Wang ${ }^{1,2,6}$, Huiling Guo ${ }^{1,6}$, Yin-Yue Zhao ${ }^{1}$, Hui-Hui Sun${ }^{1}$, Yi-Fan Li ${ }^{1}$, Xiao-Ying Lai ${ }^{1}$, Ning Zhao', Xu Wang ${ }^{2}$, Changchuan Xie', Lixin Hong ${ }^{1}$, Xi Huang ${ }^{1}$, Hong-Rui Wang (1,3, Cheng-Bin Li ${ }^{4}$, Bin Liang ${ }^{4}$, Shuai Chen ${ }^{5 凶}$ \& Tong-Jin Zhao (1) $1,2,3 \bowtie$

Fatty acids (FAs) are essential nutrients, but how they are transported into cells remains unclear. Here, we show that FAs trigger caveolae-dependent CD36 internalization, which in turn delivers FAs into adipocytes. During the process, binding of FAs to CD36 activates its downstream kinase LYN, which phosphorylates DHHC5, the palmitoyl acyltransferase of CD36, at Tyr91 and inactivates it. CD36 then gets depalmitoylated by APT1 and recruits another tyrosine kinase SYK to phosphorylate JNK and VAVs to initiate endocytic uptake of FAs. Blocking CD36 internalization by inhibiting APT1, LYN or SYK abolishes CD36dependent FA uptake. Restricting CD36 at either palmitoylated or depalmitoylated state eliminates its FA uptake activity, indicating an essential role of dynamic palmitoylation of CD36. Furthermore, blocking endocytosis by targeting LYN or SYK inhibits CD36-dependent lipid droplet growth in adipocytes and high-fat-diet induced weight gain in mice. Our study has uncovered a dynamic palmitoylation-regulated endocytic pathway to take up FAs.

\footnotetext{
${ }^{1}$ State Key Laboratory of Cellular Stress Biology, School of Life Sciences, Xiamen University, Xiamen 361102 Fujian, China. ${ }^{2}$ Institute of Metabolism and Integrative Biology, Fudan University, Shanghai 200438, China. ${ }^{3}$ State-Province Joint Engineering Laboratory of Targeted Drugs from Natural Products, Xiamen University, Xiamen 361102 Fujian, China. ${ }^{4}$ Center for Life Sciences, School of Life Sciences, Yunnan University, Kunming 650091, China. ${ }^{5}$ State Key Laboratory of Pharmaceutical Biotechnology, Model Animal Research Center, Nanjing Biomedical Research Institute, Nanjing University, Pukou District, Nanjing 210061, China. ${ }^{6}$ These authors contributed equally: Jian-Wei Hao, Juan Wang, Huiling Guo. ${ }^{凶}$ email: chenshuai@nju.edu.cn; zhaotj@fudan.edu.cn
} 


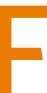
atty acids (FAs) exert multiple functional roles, including serving as an energy source and as precursors for membrane synthesis and energy storage ${ }^{1}$. FAs and their derivatives can also function as signaling molecules to regulate multiple cellular activities. Despite the important roles of FAs, the first regulatory step for their utilization, i.e. transporting FAs across the plasma membrane, is not well understood.

Unlike glucose and amino acids, FAs are hydrophobic and have extremely low aqueous solubility at physiological pH7.4, and they are usually bound with serum albumins and cytoplasmic FA binding proteins ${ }^{2}$. The hydrophobicity makes it difficult to track the movement of FAs. It was proposed that passive diffusion might be the predominant way to absorb $\mathrm{FAs}^{3}$, but accumulating evidences support that protein-facilitated FA uptake is the key pathway in metabolic tissues including liver, adipose tissue, and muscle ${ }^{4,5}$

Among the proteins involved in FA uptake, CD36 is a major player in metabolic tissues ${ }^{6,7}$. It is primarily localized in and requires caveolae for its FA uptake activity ${ }^{8-10}$. CD36 accounts for $50 \%$ of the FA uptake in adipose tissues and muscle in mice ${ }^{11}$. Humans with CD36 deficiency also show significantly decreased FA uptake in heart ${ }^{12}$, muscle, and adipose tissues ${ }^{13}$. CD36 has an FA binding pocket ${ }^{14}$, and it was proposed to be a transporter ${ }^{6}$; however, a study using HEK293 cells shows that CD36 increases intracellular esterification but not translocation of FAs ${ }^{15}$. It still remains unknown about how exactly CD36 transports FAs across the plasma membrane.

Protein palmitoylation is catalyzed by a group of Asp-HisHis-Cys (DHHC)-motif containing palmitoyl acyltransferases (DHHCs) by attaching a palmitoyl group to a thiol group of a cysteine residue ${ }^{16,17}$. Dynamic palmitoylation provides a key regulatory mechanism for subcellular localization and intracellular trafficking of proteins ${ }^{18,19}$. We have previously shown that palmitoylation of CD36 by DHHC4 and DHHC5 is required for its plasma membrane localization and FA uptake activity $^{20}$. DHHC4 palmitoylates CD36 at Golgi and creates a sorting signal, and DHHC5 maintains CD36 at the cell surface by protecting it from depalmitoylation ${ }^{20}$. It is unknown about whether and how the palmitoylation of CD36 is dynamically regulated.

Endocytosis is an essential way to transport materials, especially large molecules or those hard to penetrate the plasma membrane, into cells ${ }^{21}$. Here, we report that FAs can be transported into cells by CD36-mediated endocytosis. We figure out the signaling pathway and the machineries required for the process, and identify dynamic palmitoylation of CD36 as a key regulatory mechanism. Restricting CD36 either at palmitoylated or depalmitoylated state abolishes its FA uptake activity. Importantly, blocking the endocytosis significantly inhibits CD36-dependent FA uptake, lipid droplet growth, and weight gain on high-fat diet (HFD). We have thus uncovered CD36-mediated endocytosis as a physiologically important pathway to deliver FAs and provided valuable insights on how proteins facilitate the transport of FAs into cells.

\section{Results}

Long-chain FAs trigger internalization of CD36. To study changes in subcellular localization of CD36 during FA uptake, we treated 3T3-L1 adipocytes with BSA-conjugated oleate for various time, and performed immunostaining of endogenous CD36 in a 3-dimensional (3D) mode. CD36 stayed on the plasma membrane up to $15 \mathrm{~min}$ after oleate treatment. Starting from $30 \mathrm{~min}$, CD36 was internalized and displayed intracellular puncta-like signals inside the cells. By $1 \mathrm{~h}$, CD36 started to wrap around lipid droplets, which became more obvious at 2 and $4 \mathrm{~h}$ (Fig. 1a and Supplementary Movies 1-6). As CD36 is primarily localized in the caveolae structures $^{8}$, we have also examined the dynamic localization of Caveolin1 (CAV1), a scaffold protein of caveolae, and found that CAV1 co-migrated with CD36 (Fig. 1a and Supplementary Movies 1-6). To better illustrate the internalization of CD36, we checked the colocalization of CD36 with ATP1A1, a subunit of $\mathrm{Na}^{+} / \mathrm{K}^{+}$-ATPase that is usually localized on the plasma membrane. As shown in Supplementary Fig. 1a, ATP1A1 remained on the plasma membrane in either BSA- or oleate-treated cells, whereas CD36 showed clear intracellular localization in oleate-treated cells. To further confirm the results, we examined the caveolae structures by electron microscopy. In BSA-treated cells, caveolaes were mainly localized on the plasma membrane; however, large amounts of caveolaes were internalized $1 \mathrm{~h}$ after oleate treatment (Supplementary Fig. 1b). Furthermore, we performed surface biotinylation assay to examine the plasma membrane content of CD36 and ATP1A1. As Triton X-100 used in the assay would not disrupt the caveolae structures, capture of biotinylated CD36 could also pull down CAV1, and we therefore detected CAV1 at the same time. Consistently, the plasma membrane content of ATP1A1 did not change much before and after oleate treatment, but the plasma membrane content of CD36 and CAV1 significantly decreased with time (Fig. 1b and Supplementary Fig. 1c).

Considering that the FA binding pocket of CD36 is on the outer layer of plasma membrane and CAV1 on the inner side, we wondered whether CD36 and CAV1 would depend on each other for the internalization in a way that CD36 acts like a receptor to receive the signal from FAs and CAV1 provides structural support. Indeed, when either one of them was knocked down in 3T3-L1 adipocytes, the other one would no longer get internalized after oleate treatment (Fig. 1c), which is further confirmed by surface biotinylation assay and quantification of surface CD36 content (Fig. 1d and Supplementary Fig. 1e), suggesting that the internalization of them is CD36mediated caveolar endocytosis.

To confirm that the internalization is triggered by the binding of FAs to CD36, we generated the K164R mutant of CD36 which lacks FA binding activity ${ }^{22}$, and electroporated WT CD36 or K164R into the adipocytes derived from stromal vascular fractions (SVFs) isolated from inguinal white adipose tissue (iWAT) of $C d 36^{-1-}$ mice. As expected, WT CD36 was localized on the plasma membrane in BSA-treated cells, and internalized upon oleate treatment. In contrast, K164R did not respond to oleate and stayed on the plasma membrane in both BSA- and oleate-treated cells (Supplementary Fig. 1d).

To test whether all long-chain FAs could trigger the endocytosis, we treated 3T3-L1 adipocytes with FAs possessing different chain lengths or saturation including myristate $(\mathrm{C} 14: 0)$, palmitate $(\mathrm{C} 16: 0)$, stearate (C18:0), linoleate (C18:2), and arachidonate (C20:4), and found that all FAs were able to trigger internalization of CD36 (Fig. 1e). The results were further confirmed by surface biotinylation and quantification of the surface content of CD36 (Fig. If and Supplementary Fig. 1f).

We then examined whether CD36 would be recycled to the plasma membrane after withdrawal of FAs from the medium. As shown in Fig. 1g, 30 min after removal of oleate, a small fraction of CD36 started to relocate to the plasma membrane. By $1 \mathrm{~h}$, the majority of CD36 was recycled to the plasma membrane. And by $4 \mathrm{~h}$, almost all CD36 was observed on the plasma membrane. These results were further confirmed by surface biotinylation assay (Fig. 1h and Supplementary Fig. 1g).

CD36-mediated endocytosis delivers FAs into cells. As CD36 binds FAs ${ }^{14}$, we wondered whether the FA-triggered endocytosis was to deliver FAs into cells. We chose PacFA as an FA analog to 
a

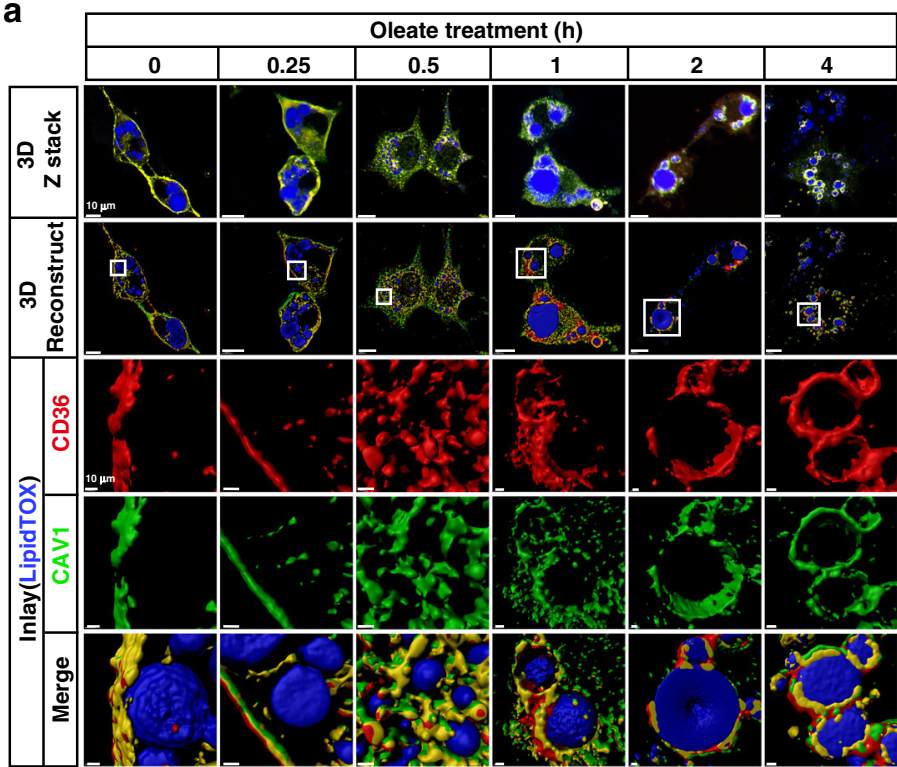

C
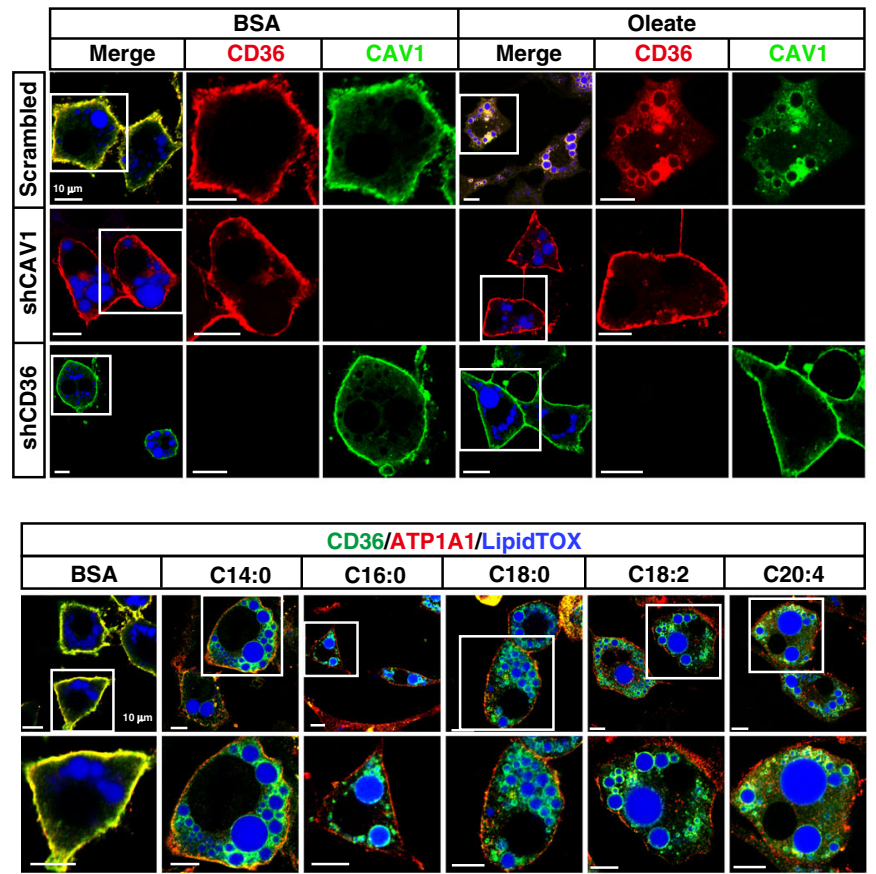

g

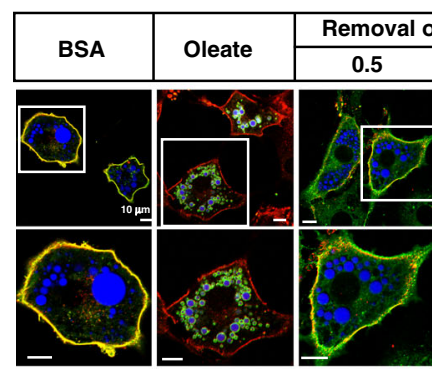

\begin{tabular}{|c|c|c|c|}
\hline of Oleate (h) & \multicolumn{2}{c}{ CD36/ATP1A1/LipidTOX } \\
\hline 1 & 2 & 4 \\
\hline
\end{tabular}

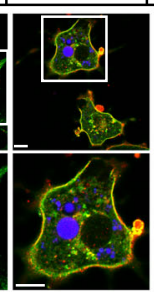

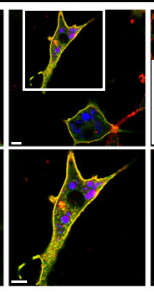

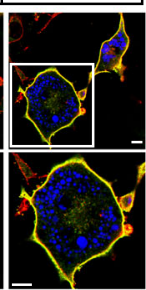

b

\begin{tabular}{|l|l|l|l|l|l|l|}
\hline Oleate (h) & 0 & 0.25 & 0.5 & 1 & 2 & 4 \\
\hline
\end{tabular}
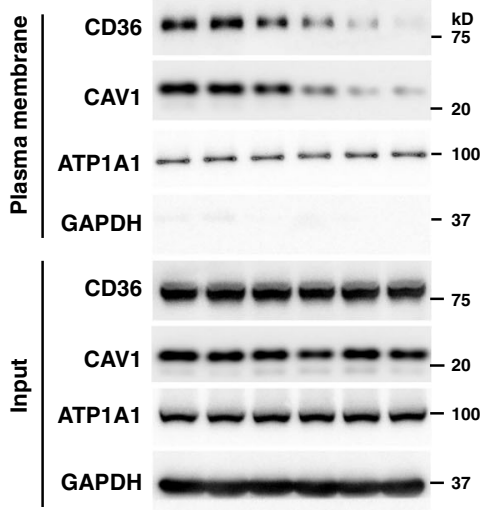

d \begin{tabular}{|c|c|c|c|}
\hline ShRNA & Scr. & ShCD36 & ShCAV1 \\
\hline Oleat & $-1+t$ & $-1+t$ & $-1+t$ \\
\hline
\end{tabular}

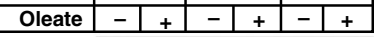

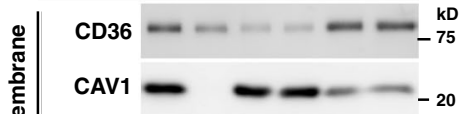

ATP1A1 - - - - - 100

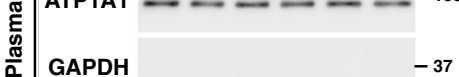

CD36 ------75

홀

ATP1A1 - - - - -100

CAV1 $------_{-20}$

GAPDH - -37

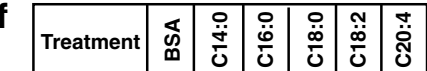

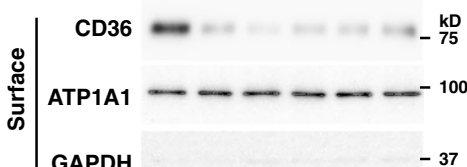

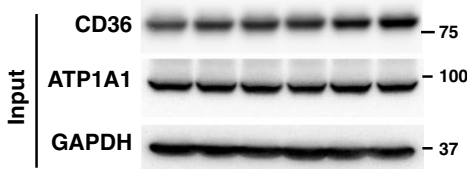

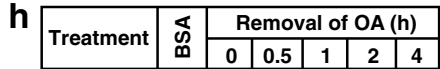

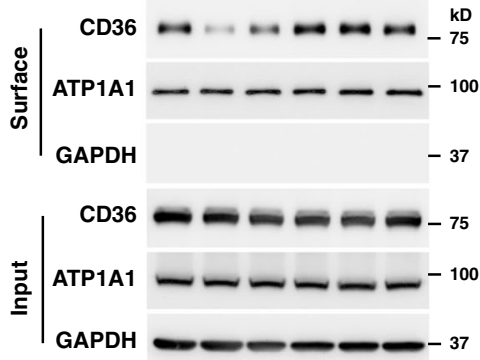

track the movement of FAs in the cells. As illustrated in Fig. 2a, PacFA is a photoactivable and clickable FA analog, and it is able to get incorporated into triglycerides, phospholipids, and cholesterol esters ${ }^{23}$. We treated 3T3-L1 adipocytes with PacFA for $20 \mathrm{~min}$ when the endocytosis just started, aiming at trapping the complex of PacFA and CD36 in the endocytosed caveolaes, followed by UV crosslinking, click chemistry with $\mathrm{N}_{3}$-Alexa Fluor
488 to label PacFA, and immunostaining of CD36. Indeed, a clear localization of PacFA and CD36 was observed (Fig. 2a). When quantified, about $56 \%$ of PacFA was bound with CD36 inside the cells (Fig. 2b).

To further confirm the results, we pulled down PacFA-bound proteins by using $\mathrm{N}_{3}$-biotin for click chemistry. As shown in Fig. 2c, capture of PacFA by streptavidin beads specifically pulled 
Fig. 1 FAs trigger internalization of CD36. $\mathbf{a}$, $\mathbf{b}$ On day 8 of differentiation, 3T3-L1 adipocytes were pretreated with serum-free medium for $4 \mathrm{~h}$, and then treated with BSA-conjugated oleate $(100 \mu \mathrm{M})$ for indicated time. a One set of cells was subjected to immunostaining with anti-CD36 and anti-CAV1 antibodies. LipidTOX was used to label lipid droplets. Images were taken under a Zeiss LSM-780 microscope in a 3D Z-stack mode and reconstructed using Imaris 9.2.0. b The other set of cells was subjected to surface biotinylation assay and blotted with indicated antibodies. c, d On day 4 of differentiation, 3T3-L1 cells were infected with lentivirus encoding scrambled shRNA or shRNAs against CD36 or CAV1. On day 5, cells were selected with $5 \mu \mathrm{g} / \mathrm{ml}$ puromycin. On day 8 , cells were pretreated as in (a) and treated with oleate $(100 \mu \mathrm{M})$ for $4 \mathrm{~h}$, followed by immunostaining with anti-CD36 and anti-CAV1 antibodies (c), or surface biotinylation assay (d). e, f 3T3-L1 adipocytes were pretreated as in (a) and treated with BSA-conjugated FAs with different chain lengths or saturation $(100 \mu \mathrm{M})$ for $4 \mathrm{~h}$. Cells were subjected to immunostaining with anti-CD36 antibody (e), or surface biotinylation assay (f). After oleate treatment for $4 \mathrm{~h}, 3 \mathrm{T3}$-L1 adipocytes were switched to serum-free medium for indicated time and harvested for immunostaining (g) and surface biotinylation $(\mathbf{h})$. The scale bars were as indicated in each figure. These experiments were repeated at least three times. Source data are provided as a Source Data file.

a

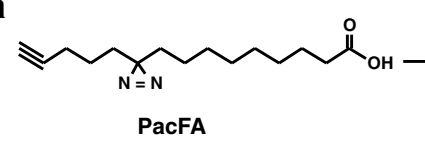

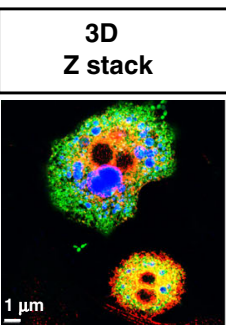

\begin{tabular}{|c|c|}
\hline $\begin{array}{c}\text { 3D } \\
\text { Reconstruct }\end{array}$ \\
\hline
\end{tabular}

C

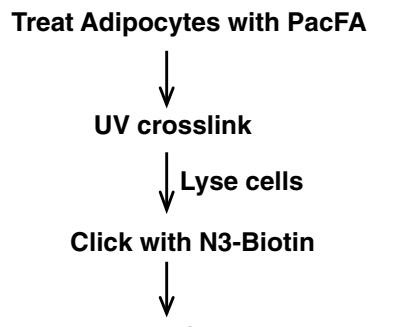

Pull down with Streptavidin beads

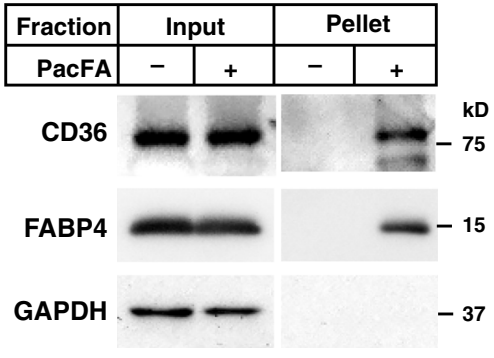

b
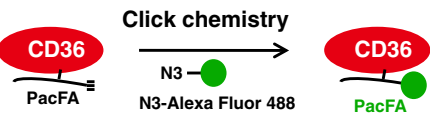

Adipocyte

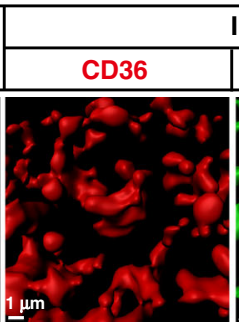

Inlay(LipidTOX)

PacFA-488
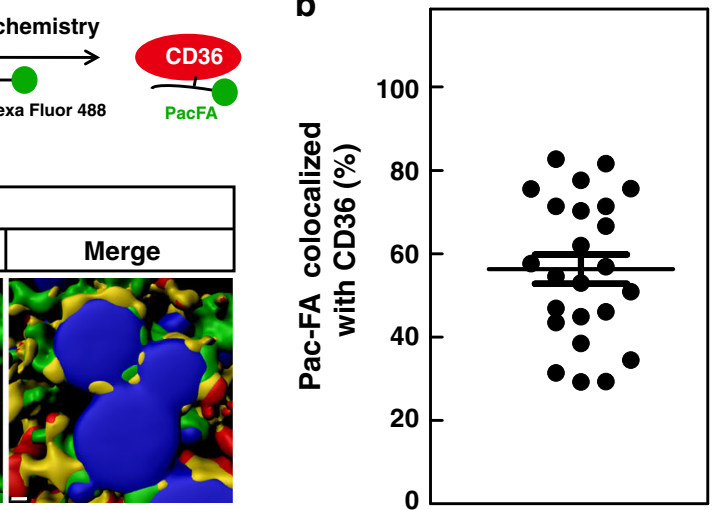

d

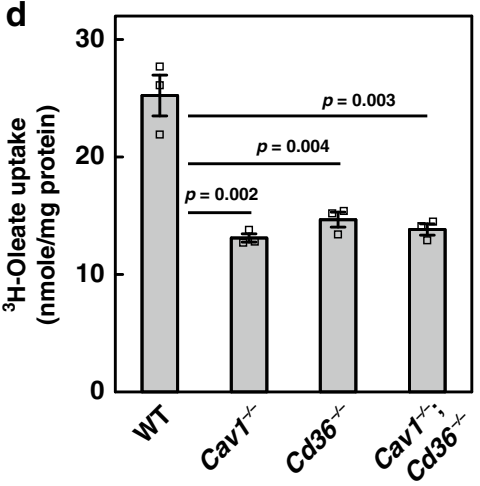

Fig. 2 CD36-mediated caveolar endocytosis transports FAs into cells. a-c 3T3-L1 adipocytes were pretreated with serum-free medium for $4 \mathrm{~h}$, and then treated with BSA-conjugated PacFA $(50 \mu \mathrm{M})$ for 20 min, followed by UV crosslinking on ice for 30 min. $\mathbf{a}, \mathbf{b}$ Cells were subjected to click chemistry using an $\mathrm{N}_{3}$-Alexa Fluro 488, and immunostained with anti-CD36 antibody. Colocalization of PacFA and CD36 was quantified from 24 cells over three independent experiments and plotted in (b). The value represents mean \pm SEM. c Cells were lysed and subjected to click chemistry assay using $\mathrm{N}_{3}$-biotin. PacFA-labeled proteins were captured with streptavidin beads and subjected to western blot using anti-CD36 and anti-FABP4 antibodies. d WT, Cav1 ${ }^{-/-}$,

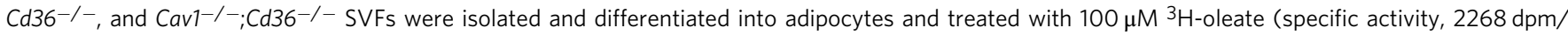
$\mathrm{nmol}$ ) for $1 \mathrm{~h}$. Lipid fractions were extracted from the cells and subjected to scintillation counting. The radioactive counting was normalized to protein content. Each value represents mean \pm SEM obtained from three samples. Two-sided Student's $t$ test was performed between WT and each of the knockout cells. These experiments were repeated twice. Source data are provided as a Source Data file.

down CD36. FABP4 was used as a positive control and as expected it was also pulled down by PacFA. These results indicate that CD36 physically delivers FAs inside the cells.

To further confirm that CD36 facilitates FA uptake by endocytosis, we examined whether blocking its endocytosis by depleting CAV1 would abolish its FA uptake. We performed ${ }^{3} \mathrm{H}$-oleate uptake in adipocytes derived from SVFs of $\mathrm{Cav1}^{-1-}$, $\mathrm{Cd} 36^{-I-}$, and $\mathrm{Cav1} 1^{-I-} ; \mathrm{Cd} 36^{-1-}$ mice. Indeed, similar to $C d 36^{-1-}$ adipocytes, Cav1-l- adipocytes showed a $40 \%$ decrease in ${ }^{3} \mathrm{H}$-oleate uptake, when compared with WT adipocytes (Fig. 2d). Double knockout of Cav1 and Cd36 did not further decrease ${ }^{3} \mathrm{H}$-oleate uptake, indicating that caveolar endocytosis is required for CD36-dependent FA uptake.

Dynamic palmitoylation of CD36 is required for FA uptake. We then moved on to study how CD36 was dissociated from the plasma membrane. As palmitoylation is required for the plasma membrane localization of $\mathrm{CD} 36^{20}$, we therefore wondered whether CD36 would get depalmitoylated before internalization. We checked changes in the palmitoylation levels of CD36 after various time of oleate treatment. As shown in Fig. 3a, palmitoylation of $\mathrm{CD} 36$ started to decrease even $5 \mathrm{~min}$ after oleate 


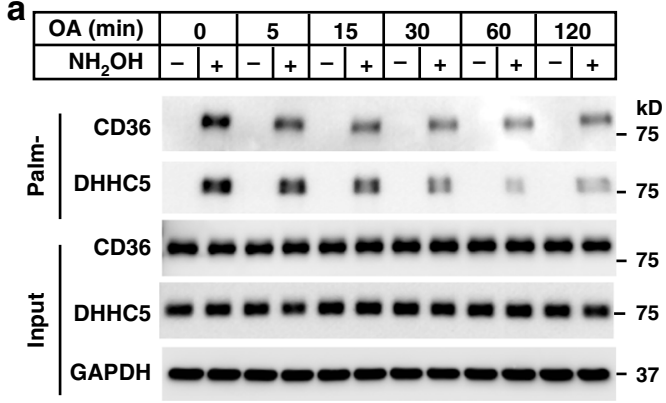

C

\begin{tabular}{|c|c|c|c|c|c|c|c|c|c|c|c|c|}
\hline Fraction & \multicolumn{6}{|c|}{ Input } & \multicolumn{6}{|c|}{ Surface } \\
\hline shAPT1 & \multicolumn{2}{|c|}{ Scr. } & \multicolumn{2}{|c|}{ Sh1 } & \multicolumn{2}{|c|}{ Sh2 } & \multicolumn{2}{|c|}{ Scr. } & \multicolumn{2}{|c|}{ Sh1 } & \multicolumn{2}{|c|}{ Sh2 } \\
\hline Oleate & - & + & - & + & - & + & - & + & - & + & - & + \\
\hline
\end{tabular}

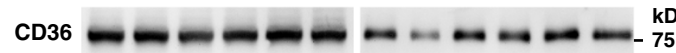

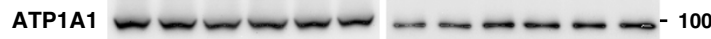

APT1 $\rightarrow--\cdots$

GAPDH

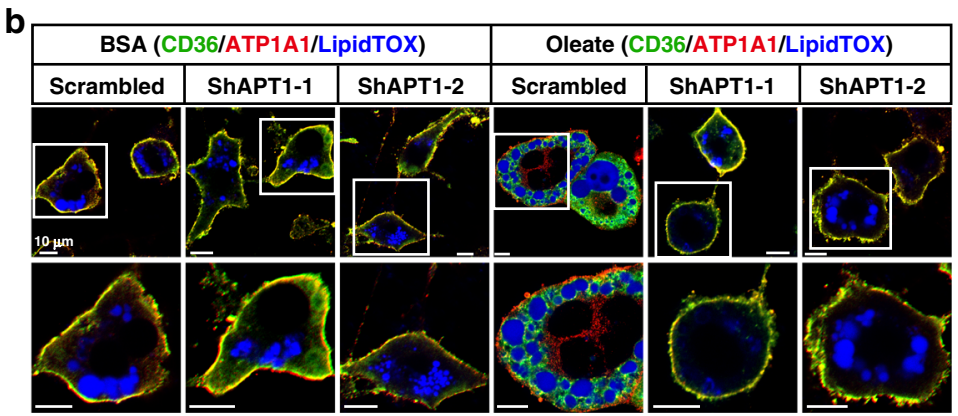

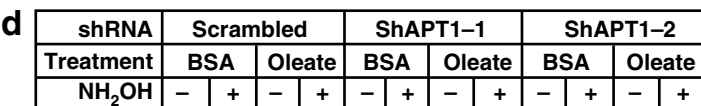

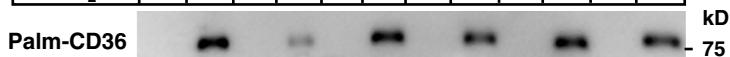

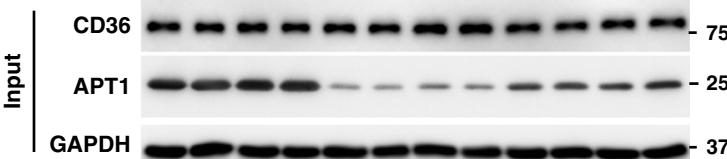

$\mathbf{e}$

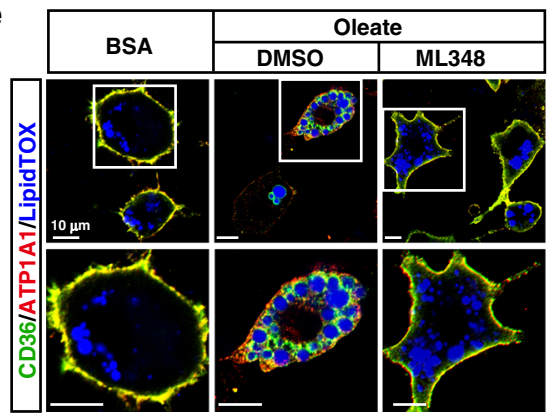

h

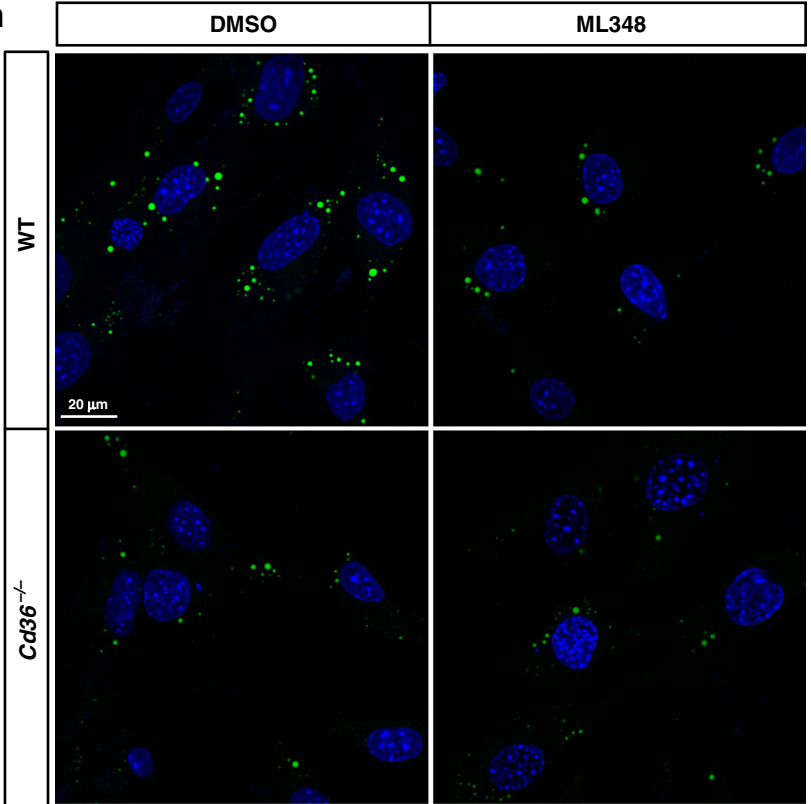

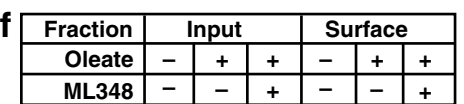

CD36

ATP1A

GAPDH

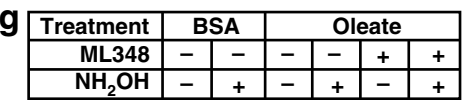

\begin{tabular}{l|l|l|l|l|l|l|}
$\mathrm{NH}_{2} \mathrm{OH}$ & - & + & - & + & - & + \\
\hline
\end{tabular}

Palm-CD36 $\rightarrow-75$

흔

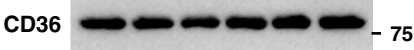

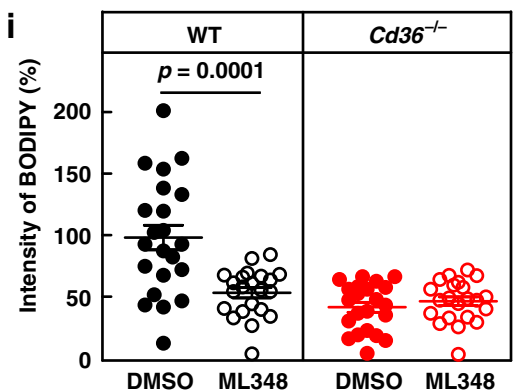

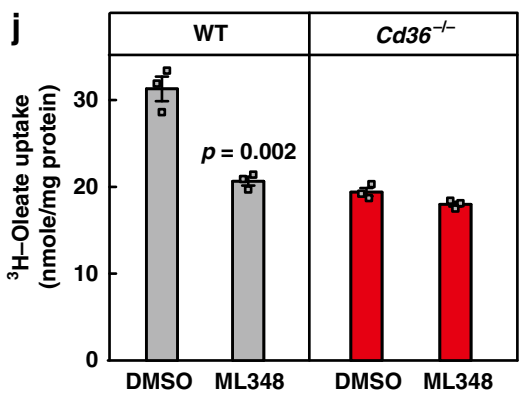

treatment, and reached nadir around 30-60 min. Similar observation was made in the palmitoylation of DHHC5, the enzyme that protects CD36 from depalmitoylation ${ }^{20}$, but there was no change in palmitoylation of CAV1 (Supplementary Fig. 2a). Consistently, when oleate was removed from the medium, CD36 and DHHC5 got re-palmitoylated (Supplementary Fig. 2b), suggesting that CD36 and DHHC5 go through dynamic palmitoylation during the FA-induced endocytosis.

To examine whether depalmitoylation of CD36 is required for the endocytosis, we treated 3T3-L1 adipocytes with palmostatin B (PalmB), a general inhibitor of depalmitoylation, and found that oleate-induced internalization of CD36 was blocked 
Fig. 3 Depalmitoylation of CD36 is required for the endocytosis of FAs. a 3T3-L1 adipocytes were pretreated and treated with oleate (100 $\mu \mathrm{M})$ as in Fig. 1a for indicated time. Cells were harvested for Acyl-RAC assay followed by immunoblotting using indicated antibodies. b-d Control (scrambled) and APT1 knockdown 3T3-L1 adipocytes were pretreated and treated with BSA or oleate $(100 \mu \mathrm{M})$ for $1 \mathrm{~h}$. Cells were then harvested for immunostaining (b), surface biotinylation (c), and Acyl-RAC (d) assays. e-g 3T3-L1 adipocytes were pretreated and treated with ML348 (10 $\mu$ M) for $1 \mathrm{~h}$, followed by BSA or oleate treatment for another $1 \mathrm{~h}$. Cells were then harvested for immunostaining (e), surface biotinylation (f), and Acyl-RAC (g) assays. h, i On day 0, WT and $\mathrm{Cd}^{3} 6^{-/}-$SVFs were set up at $4 \times 10^{4}$ cells per well in a six-well plate. On day 2 , cells were pretreated with serum-free medium for $4 \mathrm{~h}$, and treated with ML348 $(10 \mu \mathrm{M})$ for $1 \mathrm{~h}$, followed by treatment with oleate $(100 \mu \mathrm{M})$ and BODIPY $493 / 503(0.1 \mu \mathrm{g} / \mathrm{ml})$ for $4 \mathrm{~h}$. $\mathbf{h}$ Cells were then fixed and imaged on a Zeiss LSM-780 confocal microscope. i Quantification of BODIPY fluorescent intensity was performed using ZEN 2010 software. Each value represents mean \pm SEM fluorescent intensity per cell from 20 cells. The value in DMSO-treated WT cells was normalized to 1.0. Two-sided Student's $t$ test was performed between DMSO- and ML348-treated cells. DAPI was used to indicate the nuclei. $\mathbf{j}$ WT and Cd36-/- SVFs were differentiated into adipocytes and pretreated with ML348 $(10 \mu \mathrm{M})$ for $1 \mathrm{~h}$, followed by treatment with $100 \mu \mathrm{M}{ }^{3} \mathrm{H}$-oleate (specific activity, $2268 \mathrm{dpm} / \mathrm{nmol}$ ) for $1 \mathrm{~h}$. Lipid fractions were extracted from the cells and subjected to scintillation counting. The radioactive counting was normalized to protein content. Each value represents mean \pm SEM obtained from three samples. Two-sided Student's $t$ test was performed between DMSO- and ML348-treated cells. These experiments were repeated at least twice. Source data are provided as a Source Data file.

(Supplementary Fig. 2c-e). And as expected, PalmB indeed reversed oleate-induced depalmitoylation of CD36 (Supplementary Fig. 2f).

To further confirm the results, we went on to identify the depalmitoylase of CD36. There are mainly five depalmitoylases, including APT1, APT2, and ABHD17A-C 24,25 . We have previously shown that inhibition of the depalmitoylase(s) by PalmB restores plasma membrane localization of CD36 in DHHC5-deficient cells ${ }^{20}$. Taking advantage of this system, we knocked down each of the five depalmitoylases in DHHC5 knockdown 3T3-L1 preadipocytes and checked CD36 localization. As shown in Supplementary Fig. 3a, depletion of APT1, but not the others, restored the plasma membrane localization of CD36. Consistently, knockdown of APT1 restored palmitoylation of CD36 in DHHC5 ${ }^{-1}$ HEK293T cells (Supplementary Fig. 3b). To further confirm the results, we co-expressed each of the five depalmitoylases with CD36 in HEK293T cells, and examined their effects on the palmitoylation of CD36. As shown in Supplementary Fig. 3c-g, only APT1 caused a dramatic decrease in CD36 palmitoylation. Furthermore, overexpression of APT1 dissociated CD36 from the plasma membrane (Supplementary Fig. 3h). These results indicate that APT1 is the depalmitoylase of CD36.

We then knocked down APT1 in 3T3-L1 adipocytes and found that knockdown of APT1 by two separate shRNAs blocked oleate-induced internalization and depalmitoylation of CD36 (Fig. 3b-d and Supplementary Fig. 3i). To further confirm the results, we treated 3T3-L1 adipocytes with ML348, a selective inhibitor of APT $1^{26}$. Similar to knockdown of APT1, inhibition of APT1 by ML348 blocked oleate-induced internalization and depalmitoylation of CD36 (Fig. $3 \mathrm{e}-\mathrm{g}$ and Supplementary Fig. 3j).

We then examined whether blocking endocytosis by inhibiting APT1 would abolish CD36-mediated endocytic uptake of FAs. We first performed FA uptake in primary preadipocytes isolated from WT and $C d 36^{-1-}$ mice in the presence or absence of ML348. Treatment with ML348 decreased FA uptake in WT cells by $50 \%$, similar to the effect of knocking out $C d 36$, but it did not further decrease FA uptake in $C d 36^{-1-}$ cells (Fig. $3 \mathrm{~h}, \mathrm{i}$ ). To further confirm the results, we performed ${ }^{3} \mathrm{H}$-oleate uptake in SVF-derived WT and $C d 36^{-1-}$ adipocytes, and found that ML348 also abolished CD36-dependent ${ }^{3} \mathrm{H}$-oleate uptake, which is about $35 \%$ of the activity in WT cells (Fig. $3 \mathrm{j}$ ).

DHHC5 is phosphorylated and inactivated during FA uptake. We next explored what led to depalmitoylation of CD36. The fact that DHHC5 was depalmitoylated after oleate treatment (Fig. 3a) suggested that DHHC5 might get inactivated, as autopalmitoylation of DHHCs is an initial step in catalysis of palmitoylation of the substrates ${ }^{27}$. As the protein level of DHHC5 did not change after oleate treatment (Fig. 3a), we wondered whether DHHC5 was subjected to a certain posttranslational modification. We pulled down endogenous DHHC5 in BSA- or oleate-treated cells and subjected to mass spectrometry analysis. We examined potential changes in phosphorylation sites of DHHC5, as phosphorylation is usually required for rapid signal transduction. As shown in Fig. 4a, a peptide containing phosphorylated Tyr91 was specifically detected in oleate-treated cells. Topologically, Tyr91 is located in the same cytosolic loop as the DHHC motif (Fig. 4b), and the region flanking this residue is highly conserved in zebrafish, mouse, rat, and human (Fig. 4c), which makes it a potential phosphorylation site to regulate the enzymatic activity of DHHC5.

We then generated a rabbit polyclonal antibody using a synthetic peptide of DHHC5 containing phospho-Tyr91 and studied dynamic phosphorylation of Tyr91 after oleate treatment. As shown in Fig. 4d, phosphorylation of Tyr91 dramatically increased $5 \mathrm{~min}$ after oleate treatment, and it dropped to basal level after $30 \mathrm{~min}$. Furthermore, when CD36 was knocked down, oleate-induced phosphorylation of Tyr91 was largely abolished (Fig. 4e), indicating it is a downstream event of FA binding to CD36.

To test the effect of Tyr91 phosphorylation on the enzymatic activity of DHHC5, we generated Y91E and Y91F mutants to mimic the phosphorylated and unphosphorylated DHHC5, respectively. We first examined the enzymatic activity of the mutants in palmitoylating $\mathrm{CD} 36$ by reintroducing them into $\mathrm{DHHC5}^{-1-}$ HEK293T cells. While Y91F and WT DHHC5 restored palmitoylation of CD36, Y91E could barely do it (Fig. 4f). To further confirm the results, we tested the enzymatic activity the mutants in palmitoylating Flotillin-2, which was previously shown to be a substrate of $\mathrm{DHHC}^{28}$. Consistently, Y91F had similar activity with WT DHHC5 in palmitoylating Flotillin-2, but Y91E showed much decreased activity (Supplementary Fig. 4). These results indicate that phosphorylation of Tyr91 inactivates DHHC5.

We then reintroduced WT, Y91E, or Y91F of DHHC5 into Dhhc5 $5^{-1-}$ adipocytes by electroporation, and examined CD36 localization in BSA- or oleate-treated cells. As expected, when WT DHHC5 was reintroduced, CD36 was restored to the plasma membrane in BSA-treated cells, and internalized in oleate-treated cells (Fig. 4g). When Y91E was introduced, CD36 exhibited an intracellular localization when the cells were treated with either BSA or oleate, which further confirms that phosphorylation at Tyr91 inactivates DHHC5. When Y91F was introduced, CD36 stayed on the plasma membrane under both conditions, confirming that Y91F is a constitutively active form of DHHC5. The results also indicate that dynamic inactivation of DHHC5 is required for the internalization of $\mathrm{CD} 36$. 
a

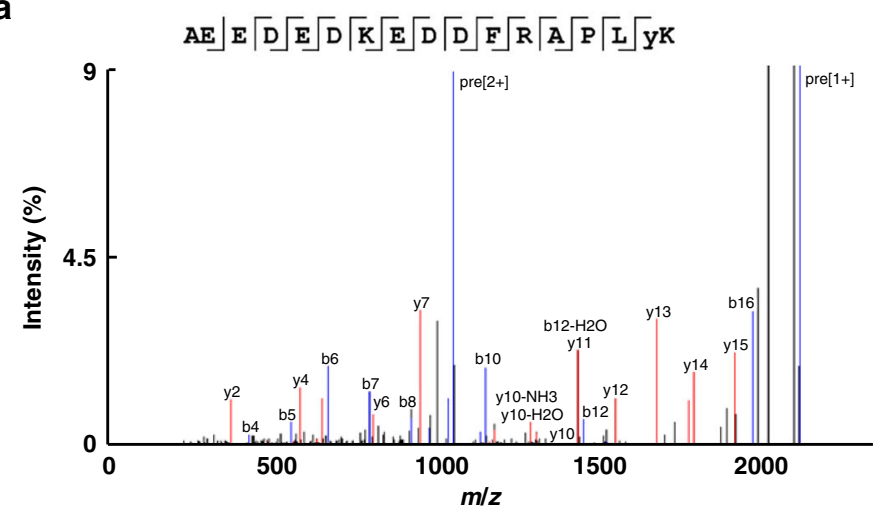

b

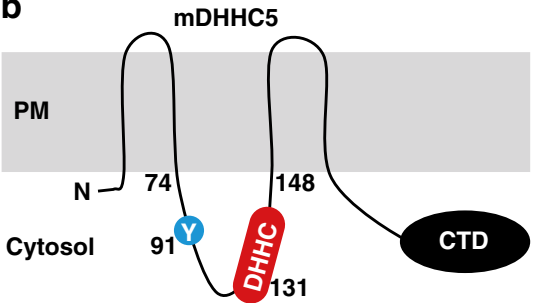

C

Human --- 83 EDDFRAPLYKTVEI 96 Mouse --- 83 EDDFRAPLYKTVEI 96 ---

Rat --- 83 EDDFRAPLYKTVEI 96 --Zebrafish --- 100 EDDFRAPLYKTVE| 113---

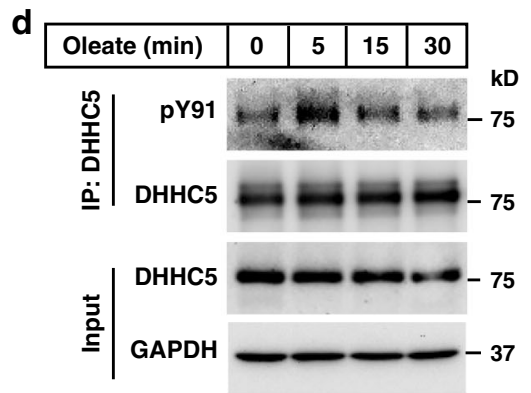

e

\begin{tabular}{|c|c|c|c|c|}
\hline shRNA & \multicolumn{2}{|c|}{ Scr. } & \multicolumn{2}{|c|}{ ShCD36 } \\
\hline Oleate & - & + & - & + \\
\hline
\end{tabular}

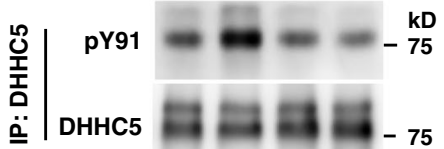

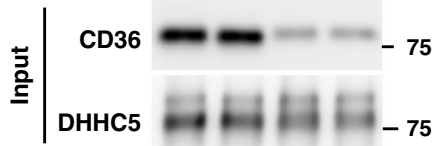

f

\begin{tabular}{|c|c|c|c|c|}
\hline Cells & \multicolumn{4}{|c|}{$\mathrm{DHHC5}^{-/-}$} \\
\hline DHHC5 & - & WT & Y91E & Y91F \\
\hline CD36 & + & + & + & + \\
\hline $\mathrm{NH}_{2} \mathrm{OH}$ & \begin{tabular}{l|l}
- & +
\end{tabular} & $-1+$ & $-1+$ & - \\
\hline
\end{tabular}

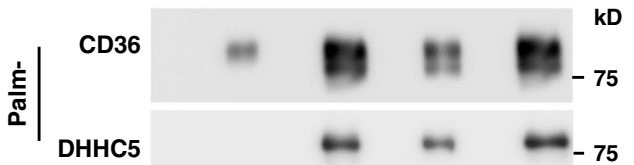

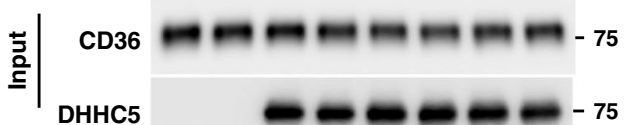
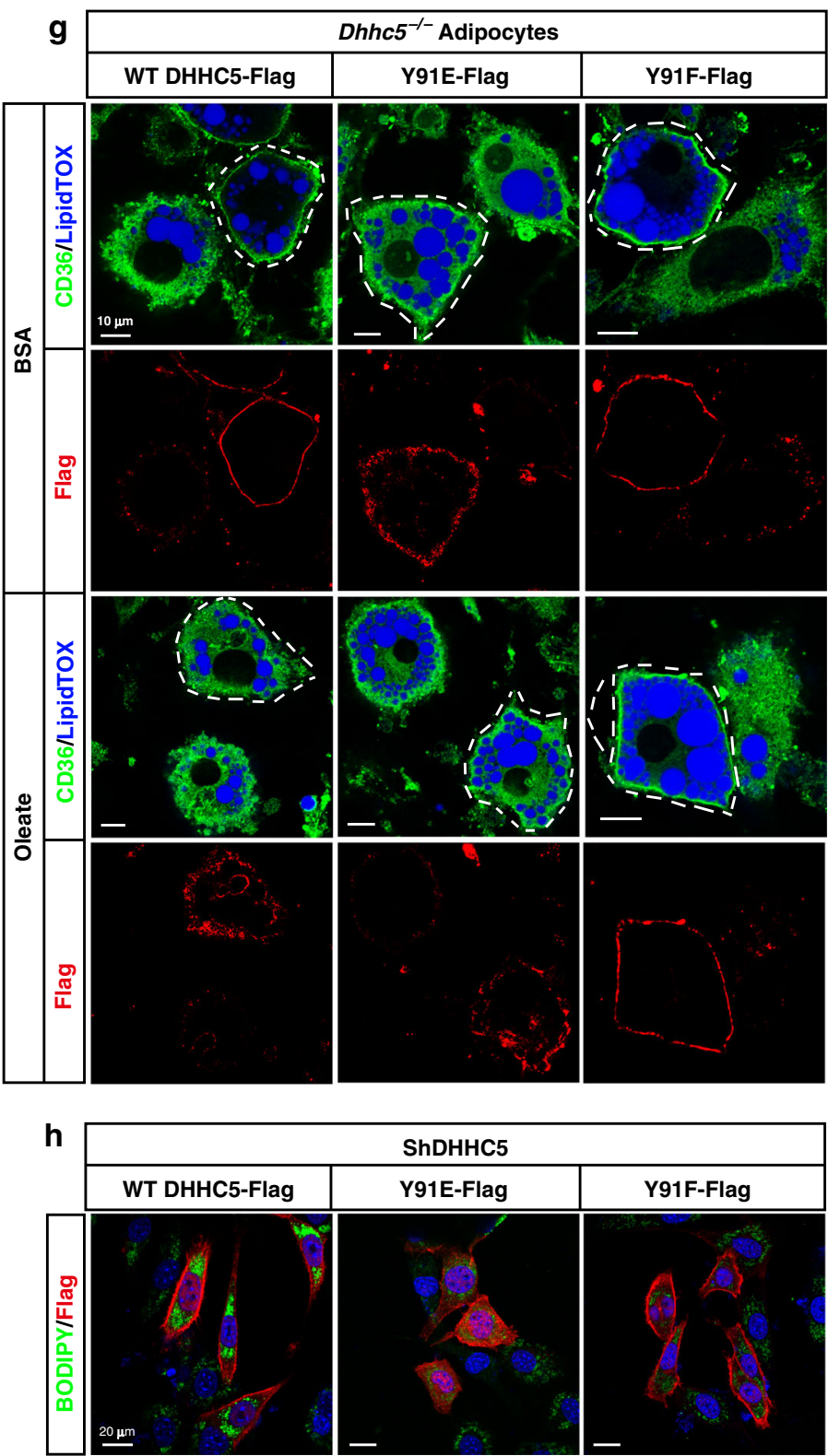

We then examined the effect of Tyr91 phosphorylation on FA uptake. We have previously shown that lack of DHHC5 abolished CD36-dependent FA uptake in 3T3-L1 preadipocytes ${ }^{20}$. We then reintroduced WT, Y91E, or Y91F into DHHC5-deficient preadipocytes and performed FA uptake. In contrast to WT DHHC5, neither Y91E nor Y91F restored the FA uptake activity in DHHC5 knockdown 3T3-L1 preadipocytes (Fig. 4h), indicating that dynamic inactivation of DHHC5 is required for the endocytic uptake of FAs.

LYN phosphorylates DHHC5 at Tyr91. We next went on to identify the kinase that phosphorylates DHHC5 at Tyr91. 
Fig. 4 Oleate triggers phosphorylation and inactivation of DHHC5. a On day 8 of differentiation, 3T3-L1 adipocytes were pretreated with serum-free medium for $4 \mathrm{~h}$, and then treated with BSA or BSA-conjugated oleate $(100 \mu \mathrm{M})$ for $15 \mathrm{~min}$. Cells were harvested and subjected to immunoprecipitation using anti-DHHC5 antibody. The bands of DHHC5 were cut out and sent for mass spectrometry analysis of the phosphorylation sites. The spectrum of pY91-containing peptide was shown. b Indication of Y91 and DHHC motif on a topology of mouse DHHC5, which was predicted at UniProt (www.uniprot.org). c Alignment of the Y91 containing regions of DHHC5 proteins from human, mouse, rat, and zebrafish. Control and CD36 knockdown 3T3-L1 adipocytes were pretreated as in $(\mathbf{a})$, and treated with oleate $(100 \mu \mathrm{M})$ for indicated time $(\mathbf{d})$ or $5 \mathrm{~min}(\mathbf{e})$. Cells were harvested, immunoprecipitated with anti-DHHC5 antibody, and immunoblotted with an anti-pY antibody to detect phosphorylation of DHHC5. f On day 0, DHHC5 $-/-$ HEK293T cells were set up at $7.5 \times 10^{5}$ cells per $6-$ $\mathrm{cm}$ dish. On day 2, cells were transfected with $0.5 \mu \mathrm{g}$ CD36-Flag/pCDH-puro and $0.5 \mu \mathrm{g}$ of indicated DHHC5 WT, Y91E, or Y91F/pCDH-puro. On day 3, cells were harvested for Acyl-RAC assay and blotted with indicated antibodies. $\mathrm{g}$ SVFs were isolated from Rosa-CreERT2;Dhhc5f/f mice, treated with 4-OHT to induce deletion of Dhhc5, and subjected to differentiation. On day 6 of differentiation, cells were electroporated with WT, Y91E, or Y91F of DHHC5-Flag. On day 8, cells pretreated and treated with oleate as in Fig. 1c. Cells were subjected to immunofluorescence using anti-CD36 and anti-Flag antibodies. LipidTOX was used to indicate lipid droplets. Cells expressing WT, Y91E, or Y91F of DHHC5 were outlined as indicated. Scale bar, $10 \mu \mathrm{m}$. $\mathbf{h}$ DHHC5 knockdown 3T3-L1 preadipocytes were transfected with WT, Y91E, or Y91F of DHHC5-Flag. Cells were treated with oleate $(100 \mu \mathrm{M})$ and BODIPY $493 / 503(0.1 \mu \mathrm{g} / \mathrm{ml})$ for $8 \mathrm{~h}$, followed by immunostaining with anti-Flag M2 antibody. DAPI was used to indicate the nuclei. These experiments were repeated at least twice. Source data are provided as a Source Data file.

Bioinformatic analysis using GPS 3.0 (http://gps.biocuckoo.org) revealed that Tyr91 might be a potential substrate of SFKs. To test the possibility, we treated adipocytes with PP2, a selective inhibitor of $\mathrm{SFKs}^{29}$, and found that oleate-induced Tyr91 phosphorylation of DHHC5 was largely suppressed (Fig. 5a). Furthermore, oleate-induced endocytosis of CD36 and depalmitoylation of CD36 and DHHC5 were also inhibited by PP2 (Supplementary Fig. 5a-d).

To search for the kinase, we knocked down each of the four relatively abundant SFKs (LYN, FYN, SRC, and YES1) in 3T3-L1 adipocytes (Supplementary Fig. 5e), and examined the effect on oleate-induced Tyr91 phosphorylation of DHHC5. While knockdown of FYN, SRC, or YES1 showed no effect, knockdown of LYN suppressed oleate-induced phosphorylation of DHHC5 at Tyr91 (Fig. 5b and Supplementary Fig. 5f). And two separate shRNAs showed similar effects (Supplementary Fig. 5g). When co-expressed in HEK293T cells, LYN phosphorylated WT DHHC5, but not Y91E or Y91F (Fig. 5c). Furthermore, knockdown of LYN with two separate shRNAs blocked oleate-induced internalization of CD36 and depalmitoylation of CD36 and DHHC5 (Fig. 5d-f and Supplementary Fig. 5h), confirming that LYN phosphorylates DHHC5 at Tyr91.

We then examined LYN activation after oleate treatment. Phosphorylation of LYN at Tyr396, which indicates the activation of LYN, was significantly increased $5 \mathrm{~min}$ after oleate treatment, and the signal dropped to basal level at $15 \mathrm{~min}$ (Fig. $5 \mathrm{~g}$ ). Furthermore, when CD36 was knocked down, oleate-induced phosphorylation at Tyr396 was largely abolished (Fig. 5h), indicating that LYN is a downstream kinase of CD36.

To further confirm the requirement of LYN in CD36-mediated endocytosis of FAs, we inhibited LYN with PP2 to block CD36 internalization and performed FA uptake. Similar to the effect of ML348, treatment with PP2 abolished CD36-dependent FA uptake in both preadipocytes and adipocytes (Fig. 5i-k).

Depalmitoylated CD36 recruits SYK to facilitate FA uptake. We then sought to explore why depalmitoylation of CD36 is required for the endocytosis. We first examined the recruitment of dynamin, VAV, and JNK, which were previously reported to participate in caveolar endocytosis or phagocytosis of oxLDL by CD36 in macrophages ${ }^{30-34}$. We treated 3T3-L1 adipocytes with the inhibitors of dynamin (Dyngo4a), VAVs (Azathioprine), JNK (SP600125), or LYN (PP2, as a positive control), and found that all inhibitors blocked oleate-induced endocytosis of CD36 (Fig. 6a, b and Supplementary Fig. 6a).

To examine whether depalmitoylation of CD36 happened before the recruitment of these factors above, we treated 3T3-L1 adipocytes with ML348 to block depalmitoylation of CD36 and examined the activation of VAV and JNK by checking their phosphorylation. As shown in Fig. 6c, oleate treatment caused increased phosphorylation of VAV (pTyr174) and JNK (pThr183/Tyr185), but such effect was largely blocked when cells were pretreated with ML348, indicating that depalmitoylation of CD36 is required for the recruitment of the machineries. These results also raised a possibility that a certain kinase might be recruited by depalmitoylated CD36 to phosphorylate VAV and JNK.

We then went on to search for the kinase. We isolated depalmitoylated CD36 by expressing CD36-Flag in $\mathrm{DHHC5^{-/- }}$ HEK293T cells and treating the cells further with 2-bromopalmitate, an inhibitor of DHHCs, to block palmitoylation of CD36. We then incubated depalmitoylated CD36-Flag with tissue lysates of gWAT prepared from mice fasted and refed with HFD, and the potential interacting proteins of depalmitoylated CD36 were analyzed by silver staining and mass spectrometry (Fig. 6d). Among the proteins, SYK, a tyrosine kinase, caused our attention, as it has been shown to be usually activated after SFKs ${ }^{35}$ and required for CD36-mediated phagocytosis of oxLDL in macrophages $^{36}$. We found that oleate treatment enhanced the interaction between CD36 and SYK (Supplementary Fig. 6b). Furthermore, SYK was activated after oleate treatment, as indicated by the phosphorylation at Tyr525/526 (Supplementary Fig. $6 \mathrm{c}$ ), and recruited to the plasma membrane (Supplementary Fig. 6d). Blockage of CD36 depalmitoylation by ML348 abolished oleate-induced phosphorylation of SYK (Fig. 6e), indicating that SYK is activated after depalmitoylation of CD36.

To examine the requirement of SYK in the endocytosis, we then treated 3T3-L1 adipocytes with piceatannol, a SYK inhibitor, and found that it largely abolished oleate-induced phosphorylation of VAV and JNK (Fig. 6f), and internalization of CD36 (Fig. 6g, h and Supplementary Fig. 6e). And piceatannol did not affect oleate-induced depalmitoylation of CD36 (Fig. 6i), confirming that SYK activation is a downstream event of CD36 depalmitoylation. Furthermore, knocking down SYK in 3T3-L1 adipocytes by two separate shRNAs blocked oleate-induced internalization of CD36 (Supplementary Fig. 6f-i).

To examine whether blocking endocytosis by inhibiting SYK would also abolish CD36-dependent FA uptake, we performed FA uptake in the presence or absence of piceatannol. Very similar to the inhibition of APT1 or LYN, piceatannol treatment also abolished CD36-dependent FA uptake in both preadipocytes and adipocytes (Fig. 6j-1).

Blocking endocytosis inhibits physiological function of CD36. We then went on to evaluate the physiological significance of CD36-mediated endocytosis of FAs. As a major physiological 

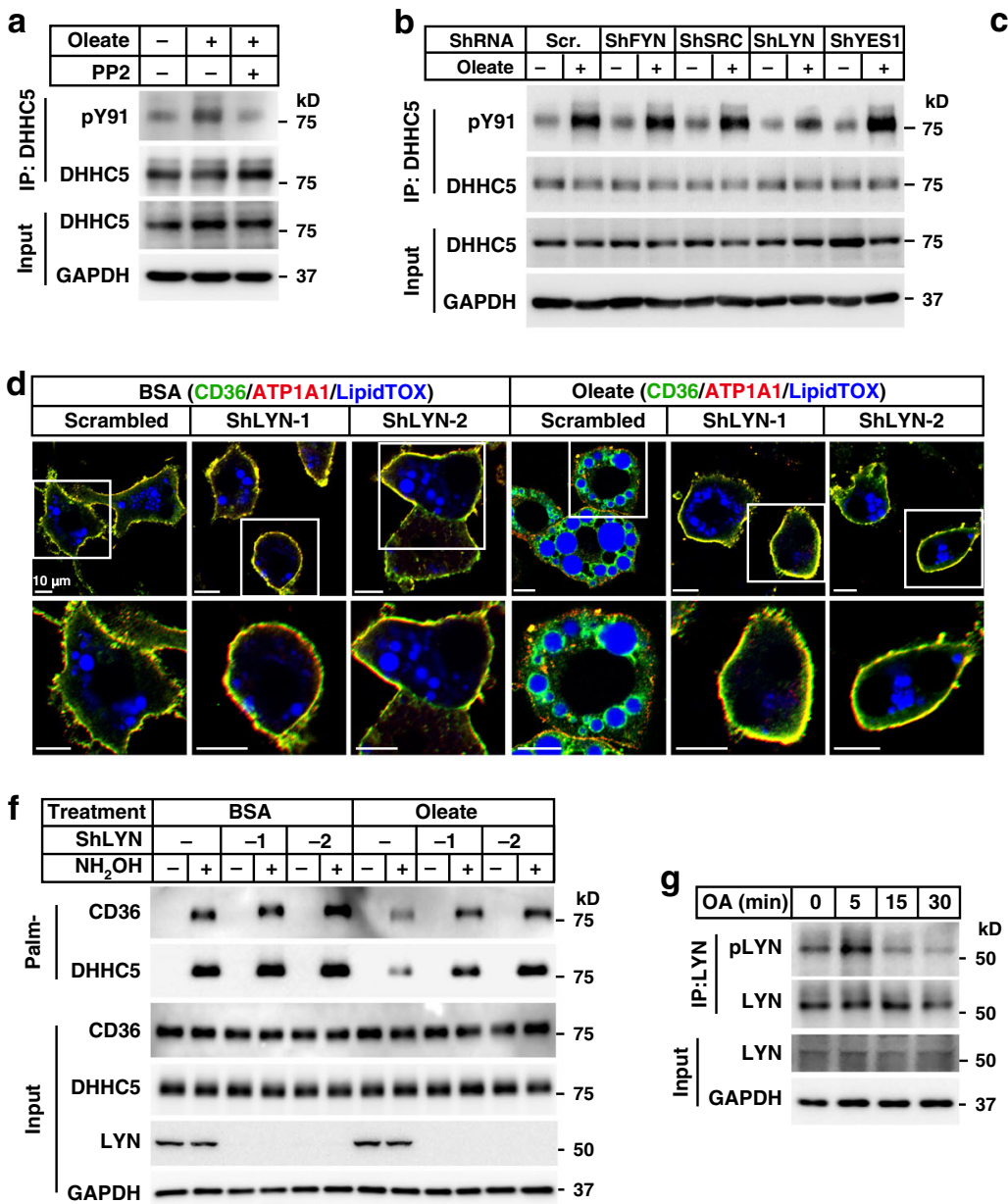

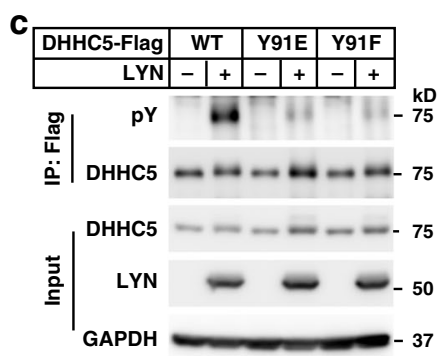

e
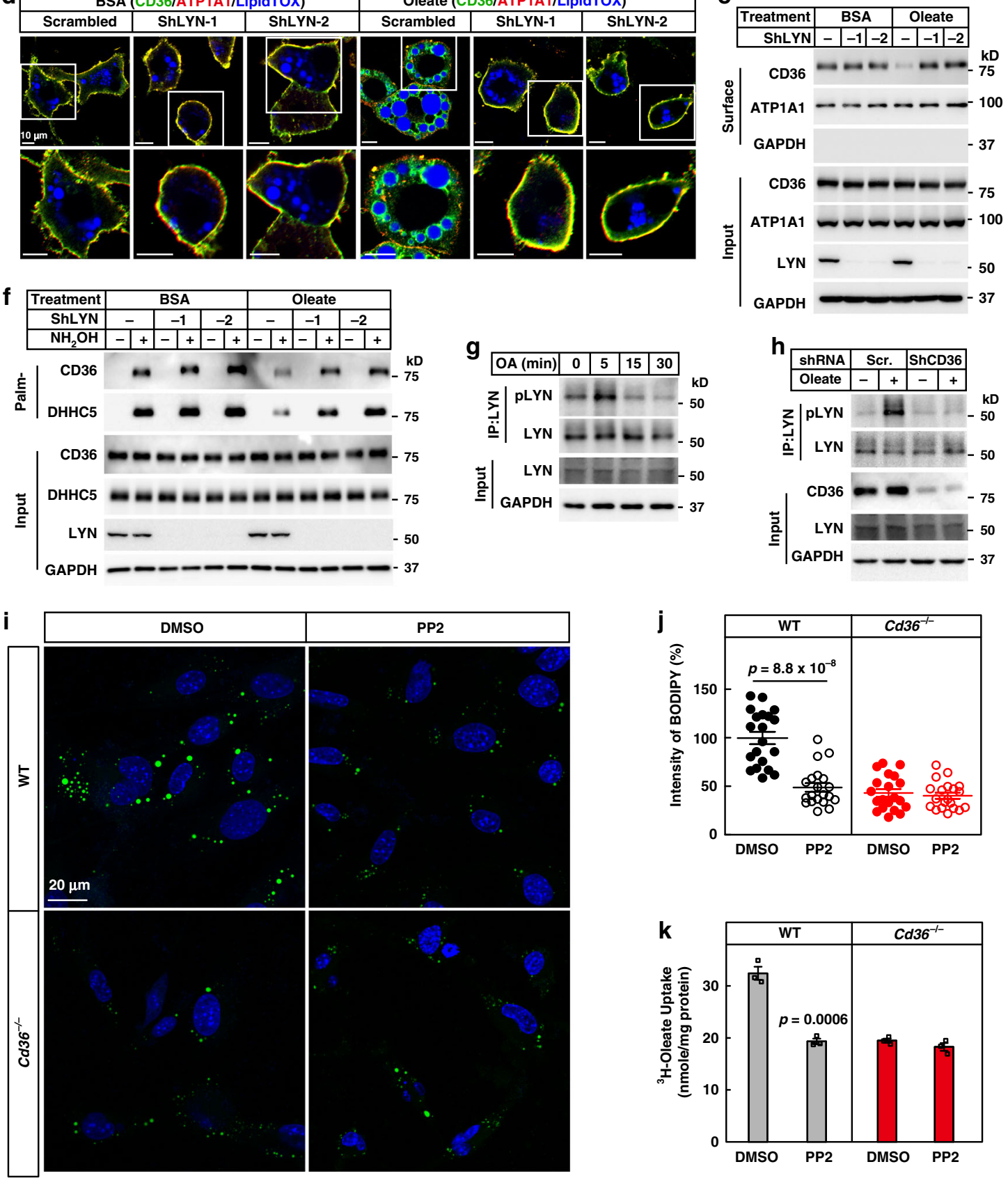

function of FA uptake is to store FAs in lipid droplets of adipocytes, we explored the effect of blocking the endocytosis on lipid droplet growth. We pretreated WT and $C d 36^{-1-}$ adipocytes with PP2, ML348, or piceatannol to inhibit LYN, APT1, or SYK, and monitored oleate-induced growth of lipid droplets using live imaging microscopy. In DMSO-treated WT adipocytes, oleate treatment promoted the growth of lipid droplets with time, and by $105 \mathrm{~min}$, the volume of lipid droplets per cell doubled (Fig. $7 \mathrm{a}$ and Supplementary Fig. 7). However, such effect of oleate was significantly suppressed when WT cells were pretreated with either PP2, ML348, or piceatannol. In DMSO-treated Cd36-1adipocytes, there was very little or no obvious growth of lipid droplets during the 2-h period. Treatment with any of the inhibitors did not further decrease lipid droplet growth (Fig. 7a and 
Fig. 5 LYN phosphorylates DHHC5 and is required for the endocytosis of FAs. a 3T3-L1 adipocytes were pretreated with serum-free medium for $4 \mathrm{~h}$ and PP2 $(15 \mu \mathrm{M})$ for $1 \mathrm{~h}$, followed by treatment with BSA or oleate $(100 \mu \mathrm{M})$ for $5 \mathrm{~min}$. Cells were harvested and immunoprecipitated with anti-DHHC5 antibody to detect phosphorylation of Tyr91. b 3T3-L1 adipocytes transduced with indicated shRNA were set up and treated with oleate for 5 min. Cells were harvested to detect Tyr91 phosphorylation as in (a). c On day 0, HEK293T cells were set up at $2.5 \times 10^{5}$ cells per 6 -cm dish. On day 2 , cells were transfected with $0.5 \mu \mathrm{g}$ of WT, Y91E, or Y91F of DHHC5-Flag/pCDH-puro and/or $0.5 \mu \mathrm{g}$ LYN/pCDNA3.3. On day 3, cells were harvested and immunoprecipitated with anti-Flag M2 beads to detect phosphorylation of DHHC5 (pY). Control and LYN knockdown adipocytes were pretreated and treated with BSA or oleate $(100 \mu \mathrm{M})$ for $1 \mathrm{~h}$, followed by immunostaining (d), surface biotinylation (e), and Acyl-RAC (f) assays. Control and CD36 knockdown 3T3-L1 adipocytes were pretreated and treated with oleate $(100 \mu \mathrm{M})$ for indicated time $(\mathbf{g})$ or 5 min (h). Cells were harvested and subjected to immunoprecipitation of LYN to detect phosphorylation of LYN (Y396). $\mathbf{i}$, j WT and Cd36-/-SVFs were set up and subjected to FA uptake as in Fig. 3h, i, except that cells were pretreated with PP2 $(15 \mu \mathrm{M})$. Each value represents mean \pm SEM obtained from 20 cells. Two-sided Student's $t$ test was performed between DMSO and PP2 treated cells. $\mathbf{k}$ WT and $\mathrm{Cd}^{3} 6^{-/-}$adipocytes were set up and subjected to ${ }^{3} \mathrm{H}$-oleate uptake as in Fig. $3 \mathrm{j}$, except that cells were pretreated with PP2 $(15 \mu \mathrm{M})$. Each value represents mean \pm SEM obtained from three samples. Two-sided Student's $t$ test was performed between DMSO and PP2 treated cells. These experiments were repeated at least twice. Source data are provided as a Source Data file.

Supplementary Fig. 7), indicating that blocking endocytosis abolished CD36-dependent lipid droplet growth in adipocytes.

We then sought to explore the physiological function of CD36mediated endocytosis of FAs on diet-induced obesity in mice. To block the endocytosis in mice, we chose bafetinib, a selective dual Bcr-ABL/LYN inhibitor ${ }^{37}$, and entospletinib, a selective SYK inhibitor $^{38}$. As expected, both compounds inhibited oleateinduced endocytosis of CD36 in 3T3-L1 adipocytes (Supplementary Fig. 8a, b). In WT mice, treatment with bafetinib or entospletinib significantly reduced weight gain on HFD (Fig. 7b). Vehicle-treated $\mathrm{Cd} 36^{-1-}$ mice gained less weight than vehicletreated WT mice, but treatment with either compound did not further decrease the body weights. We have also performed glucose tolerance test on week 8 . In WT mice, bafetinib- or entospletinib-treated mice cleared glucose much faster than vehicle-treated mice, and such effect was CD36 dependent (Supplementary Fig. 9a). We monitored food intake in the mice and found that neither bafetinib nor entospletinib changed the food intake in WT or $C d 36^{-1-}$ mice (Supplementary Fig. 9b), suggesting that the decrease in body weights of WT mice was not due to suppression of appetite. At the end of the experiment, we dissected the mice and found that treatment with bafetinib or entospletinib significantly reduced the fat mass of iWAT and gWAT, and the sizes of adipocytes in WT, but not $\mathrm{Cd} 36^{-/-}$mice (Fig. 7c, d and Supplementary Fig. 9c). To explore where the FAs were going to in bafetinib- or entospletinib-treated WT mice, we measured plasma levels of free FAs, and found that neither compound raised the plasma free FAs (Supplementary Fig. 9d). Similar observations were made in plasma triglycerides (Supplementary Fig. 9e). We then measured liver triglyceride levels and found that both compounds slightly but significantly raised liver triglyceride levels in WT mice, similar to those in the $C d 36^{-1-}$ mice (Supplementary Fig. 9f), suggesting that the decreased FA uptake activity in adipose tissues caused ectopic storage of FAs in liver.

\section{Discussion}

FAs are essential nutrients for cellular functions ${ }^{1,4}$, but how they are transported into cells remains unclear. Here, we demonstrate that long-chain FAs trigger CD36-mediated caveolar endocytosis. Using PacFA as an FA analog, we prove that FA-induced internalization of CD36 carries FAs into cells. We have figured out the key machineries and signaling pathway of the endocytic process, and shown that blocking endocytosis by depleting CAV1 or inhibiting APT1, LYN, or SYK all abolishes CD36-dependent FA uptake. Importantly, we show that the endocytic uptake of FAs is required for CD36-dependent lipid droplet growth in adipocytes and HFD-induced weight gain in mice.

It actually makes sense that CD36-mediated caveolar endocytosis represents an effective was to transport FAs into adipocytes.
First, from the point of cellular structures, caveolaes can make up to approximately one-third of the plasma membrane in adipocytes $^{39,40}$. CD36 is also highly abundant in adipocytes ${ }^{14}$, and it is primarily localized in the caveolae structures with its FA binding pocket is on the cell surface to capture FAs ${ }^{8,9}$. Such an organization ensures a large scale of caveolar endocytosis during FA uptake. Second, we show that CD36 delivers FAs to lipid droplets. Previous studies have shown that triglyceride synthesis enzymes, GPAT4, AGPAT3, and DGAT2, can relocate from ER to lipid droplets upon treatment with FAs ${ }^{41-43}$. Therefore, CD36mediated caveolar endocytosis might efficiently couples FA uptake, esterification, and triglyceride synthesis. Third, given the hydrophobic property of FAs, it might be energetically efficient to transport FAs by endocytosis, which has been adopted by other hydrophobic lipid molecules, such as $\mathrm{LDL}^{44}$, oxLDL ${ }^{45}$, and cholesterol $^{46,47}$.

Notably, we have identified a central role of dynamic palmitoylation of CD36 in regulating the endocytosis of FAs. CD36 gets depalmitoylated by treatment with FAs, and it gets re-palmitoylated after withdrawal of FAs. While palmitoylated CD36 captures FAs on the cell surface, depalmitoylated CD36 initiates the endocytic process and delivers FAs into cells. Blockage of the depalmitoylation of CD36 by depleting or inhibiting LYN or APT1 abolishes the endocytic uptake of FAs. Furthermore, restricting CD36 at palmitoylated state in Y91F DHHC5-expressing cells or depalmitoylation state in Y91E DHHC5-expressing cells abolishes the FA uptake activity of CD36. Taken together, it is the dynamic palmitoylation that ensures the FA uptake activity of CD36.

Furthermore, we have identified dynamic phosphorylation and inactivation of DHHC5 as the underlying mechanism of dynamic palmitoylation of CD36. We show that binding of FAs to CD36 activates its downstream kinase LYN, thereby converting the extracellular stimulus of FAs into intracellular signaling pathway. LYN then phosphorylates DHHC5 at Tyr91 and inactivates DHHC5, resulting in depalmitoylation of CD36 by APT1. Although there are more than 20 DHHCs in mammals 17,48 , how their enzymatic activities are regulated is barely known. Therefore, the dynamic phosphorylation by SFKs might provide a regulatory mechanism for the enzymatic activities of DHHCs family. As SFKs are widely involved in various physiological conditions ${ }^{49}$, our findings might expand the physiological functions of DHHCs to these events.

Up to date, several proteins have been reported to facilitate FA uptake, including CD36, CAV1, FATP, and FABPm ${ }^{7}$. Besides CD36, CAV1 has also been reported to get internalized after FA treatment ${ }^{50,51}$, though the physiological role was unknown. Our findings here uncover that the internalization of CD36 and CAV1 is to transport FAs into cells by CD36-mediated caveolar endocytosis. It would be interesting to see whether these other proteins would also transport FAs via endocytosis. 


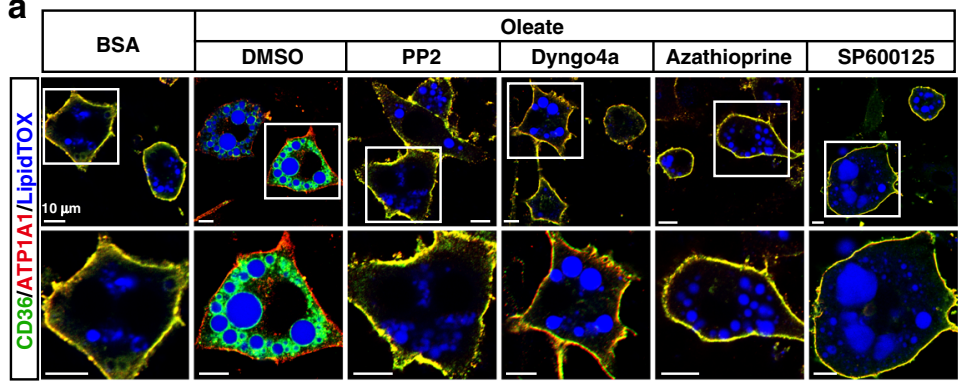

C

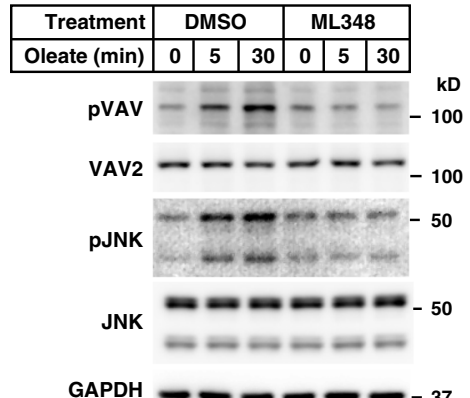

f
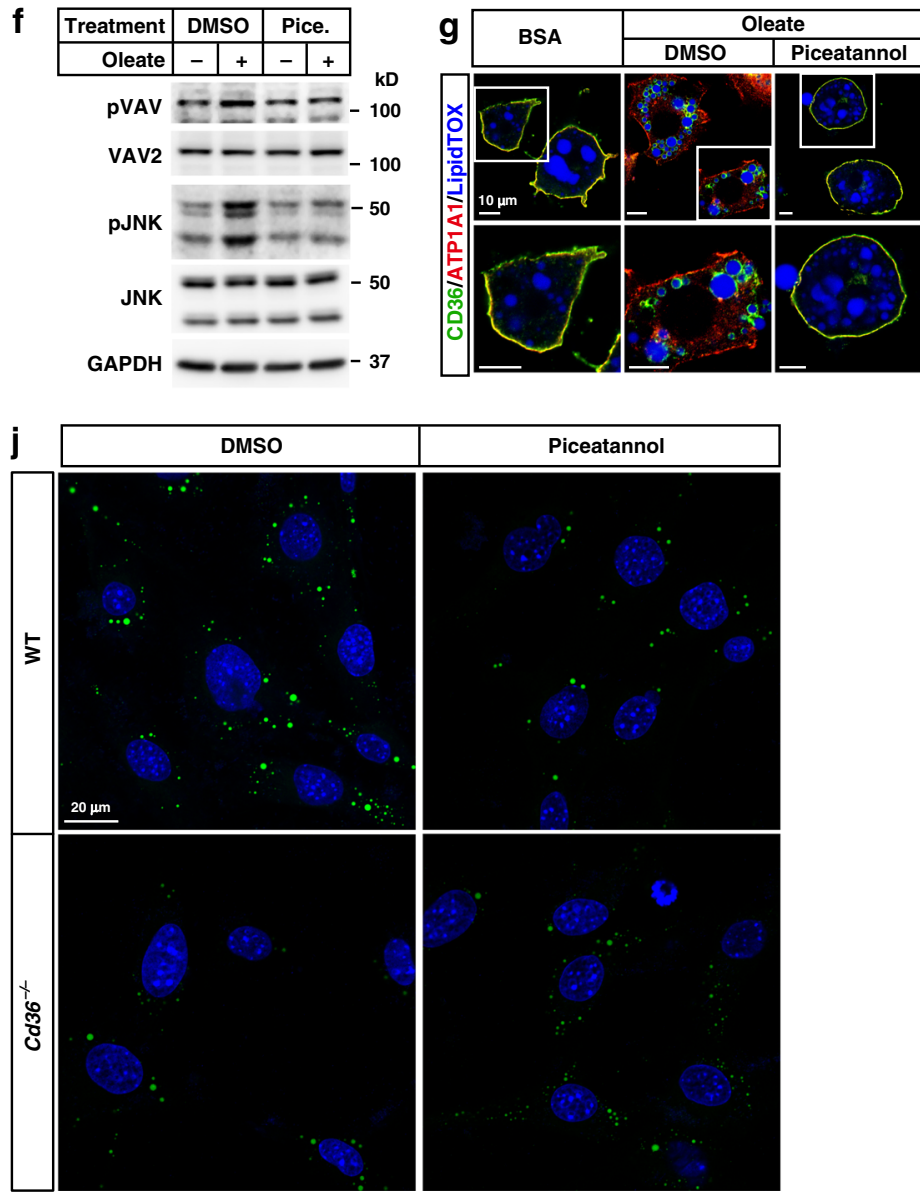

b
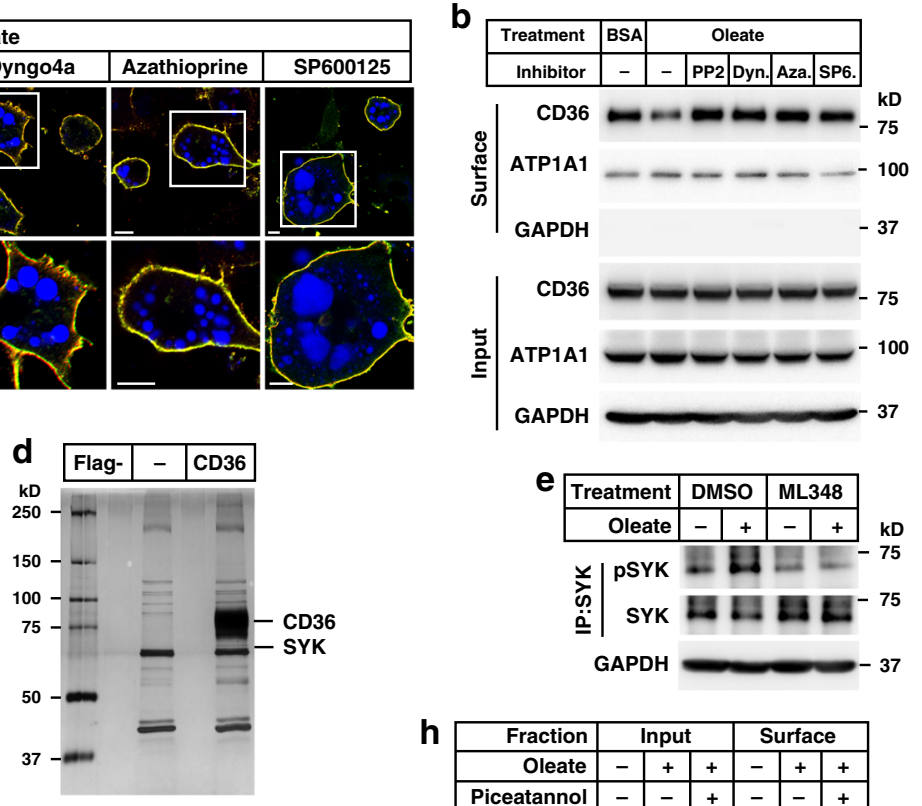

h

\begin{tabular}{|r|c|c|c|c|c|c|}
\hline Fraction & \multicolumn{2}{|c|}{ Input } & \multicolumn{3}{|c|}{ Surface } \\
\hline Oleate & - & + & + & - & + & + \\
\hline Piceatannol & - & - & + & - & - & + \\
\hline
\end{tabular}

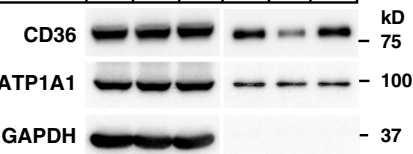

i

\begin{tabular}{|c|c|c|c|}
\hline Treatment & BSA & \multicolumn{2}{|c|}{ Oleate } \\
\hline Piceatannol & - & - & + \\
\hline $\mathrm{NH}_{2} \mathrm{OH}$ & \begin{tabular}{l|l}
-1 & +
\end{tabular} & - & - \\
\hline
\end{tabular}

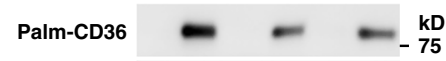

플

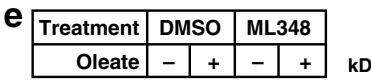

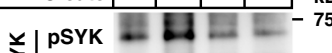

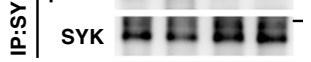
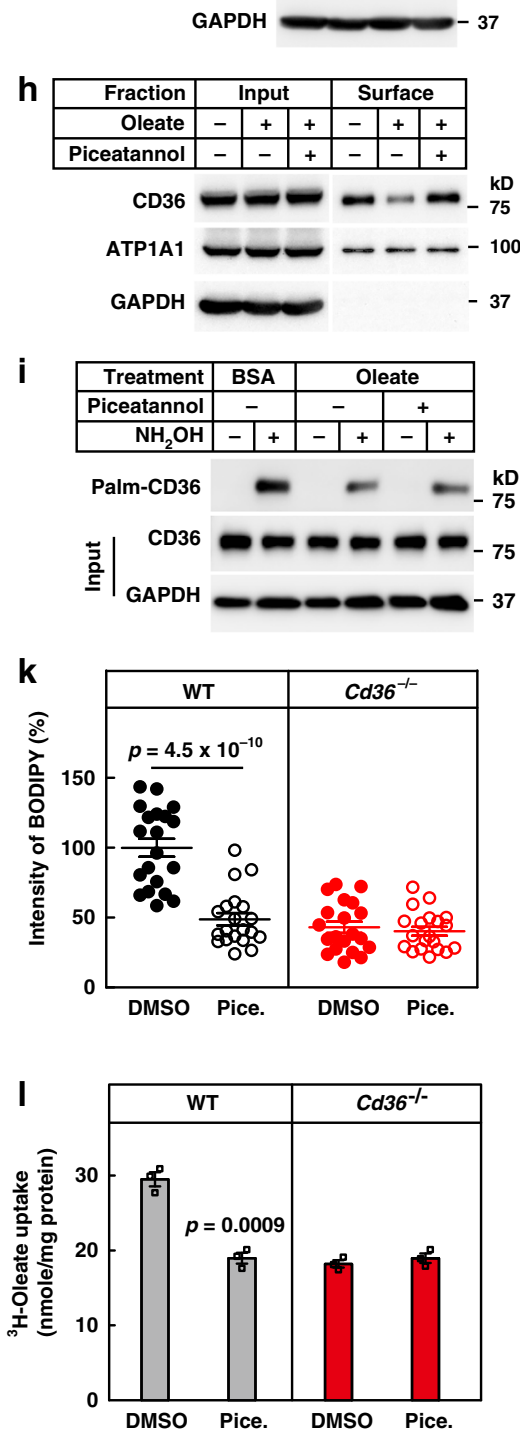

To summarize our findings, we propose a working model of the CD36-mediated endocytosis of FAs (Fig. 8). When FAs bind to CD36, its downstream kinase LYN is activated, and it phosphorylates DHHC5 at Tyr91 and inactivates DHHC5, resulting in subsequent depalmitoylation of CD36 by APT1. The depalmitoylated CD36 then recruits another tyrosine kinase, SYK, which phosphorylates VAV and JNK. VAV function as an adapter of dynamin ${ }^{32,33}$, which facilitates the pinching off of caveolaes from the plasma membrane ${ }^{21}$. The activated JNK plays an important role in regulating cytoskeleton reorganization and vesicle transport ${ }^{52}$, two key events caveolar endocytosis. Eventually, caveolaes are internalized and deliver FAs to ER for esterification and further distribution to lipid droplets for storage. After delivery, CD36 gets re-palmitoylated 
Fig. 6 Depalmitoylated CD36 recruits SYK to facilitate the endocytosis. a, b 3T3-L1 adipocytes were pretreated with serum-free medium for 4 h, and DMSO, PP2 $(20 \mu \mathrm{M})$, Dyngo4a $(2 \mu \mathrm{M})$, azathioprine $(10 \mu \mathrm{M})$, or SP600125 $(20 \mu \mathrm{M})$ for $1 \mathrm{~h}$, followed by treatment with BSA or oleate $(100 \mu \mathrm{M})$ for $1 \mathrm{~h}$. Cells were harvested for immunostaining (a) and surface biotinylation (b) assays. c 3T3-L1 adipocytes were pretreated with ML348 (10 $\mu \mathrm{M})$ for $1 \mathrm{~h}$, following by oleate treatment for 0,5 , or 30 min. Cells were harvested and immunoblotted with indicated antibodies. $\mathbf{d}$ The interacting proteins of depalmitoylated CD36 were isolated as described in "Methods." The eluted fractions were separated on SDS-PAGE, and subjected to silver staining and mass spectrometry. e 3T3-L1 adipocytes were pretreated with ML348, followed by BSA or oleate $(100 \mu M)$ for 5 min. Cells were harvested, subjected to immunoprecipitation with anti-SYK antibody, and blotted with anti-SYK and anti-pSYK (Y525/526). f 3T3-L1 adipocytes were pretreated with serum-free medium for $4 \mathrm{~h}$ and piceatannol (Pice., $40 \mu \mathrm{M}$ ) for $1 \mathrm{~h}$, followed by treatment with BSA or BSA-conjugated oleate (100 $\mu \mathrm{M})$ for 30 min. Cells were harvested for immunoblotting with indicated antibodies. $\mathbf{g}$-i 3T3-L1 adipocytes were pretreated with serum-free medium for $4 \mathrm{~h}$ and piceatannol ( $40 \mu \mathrm{M}$ ) for $1 \mathrm{~h}$, followed by treatment with BSA or BSA-conjugated oleate $(100 \mu \mathrm{M})$ for $1 \mathrm{~h}$. Cells were harvested for immunostaining (g), surface biotinylation (h), and Acyl-RAC (i) assays. j, $\mathbf{k}$ WT and $C d 36^{-/}$SVFs were set up and subjected to FA uptake as in Fig. 3h, i, except that cells were pretreated with piceatannol $(40 \mu \mathrm{M})$. Each value represents mean \pm SEM obtained from 20 cells. Two-sided Student's $t$ test was performed between DMSO and piceatannol-treated cells. I WT and $\mathrm{Cd} \mathrm{6}^{-/-}$adipocytes were set up and subjected to ${ }^{3} \mathrm{H}$-oleate uptake as in Fig. $3 \mathrm{j}$, except that cells were pretreated with piceatannol $(40 \mu \mathrm{M})$. Each value represents mean \pm SEM obtained from three samples. Two-sided Student's $t$ test was performed between DMSO and piceatannol-treated cells. The scale bars were as indicated. These experiments were repeated at least twice. Source data are provided as a Source Data file.

and recycled to the plasma membrane for another round of delivery.

\section{Methods}

Experimental models and subject details. $C d 36^{-1-}$ mice ${ }^{53}$ were purchased from Jackson laboratory, and $\mathrm{Cav1}^{-1-}$ mice were purchased from Cyagen Bioscience (KOCMP-21072-Cav1). All mice were housed in colony cages at $25^{\circ} \mathrm{C}$ with 12 -h light/12-h dark cycles, with $20-60 \%$ humidity. The dark cycle began at 7 p.m. All animal studies were performed with the approval of the Institutional Animal Care and Research Advisory Committee at Xiamen University. All mice used in the paper are male on a C57BL6 background.

Stock preparation. Stock solutions of BSA-conjugated FAs or PacFA ${ }^{23}$ (Avanti Polar Lipids, 900401P) were prepared in $0.15 \mathrm{M} \mathrm{NaCl}$ containing $10 \%$ (wt/vol) BSA (essentially FA free) at final concentrations of 5 or $10 \mathrm{mM}^{54}$. A stock solution of hydroxylamine $\mathrm{HCl}$ (2 M, Sigma-Aldrich, 159417) was freshly prepared in water and was adjusted to $\mathrm{pH} 7.5$ with $\mathrm{NaOH}$. A stock solution of azathioprine (SigmaAldrich, A4638) was prepared in $0.1 \mathrm{M} \mathrm{NaOH}$ at a final concentration of $5 \mathrm{mM}$. Stock solutions of PalmB (20 mM, Merck, 178501), PP2 (20 mM, MCE, HY13805), ML348 (10 mM, MCE, HY-100736), SP600125 (20 mM, ApexBIO, A4604), Dyngo4a (2 mM, Abcam, ab120689), and piceatannol (40 mM, MCE, HY-13518) were made up in DMSO. Bafetinib (Efebio, E074675) and entospletinib (Efebio, E012515) were prepared in $0.5 \%$ methyl cellulose in saline right before the oral gavage.

Plasmids. Full length cDNA of mouse Lyn was cloned from a cDNA library prepared from testis of a C57BL6 mouse. Human CD36 and DHHC5 were cloned in our previous studies ${ }^{20}$. These genes were cloned into either pcDNA3.3, or pCDH-EF1-MCS-IRES-Puro (System Biosciences). For knockdown, two shRNAs of each gene were designed and cloned into pLKO.1 (Addgene, 10878) ${ }^{55}$. The primer sequences are listed in Supplementary Table 1.

Differentiation of 3T3-L1 preadipocytes. 3T3-L1 preadipocytes (ATCC) were cultured in DMEM (1 g/L glucose) supplemented with $10 \%(\mathrm{v} / \mathrm{v})$ NCS (Thermal Fischer Scientific), $100 \mathrm{U} / \mathrm{ml}$ penicillin, and $100 \mathrm{mg} / \mathrm{ml}$ streptomycin at $37^{\circ} \mathrm{C}$ in an atmosphere of $8.8 \% \mathrm{CO}_{2}$. Two days after confluence, 3T3-L1 cells were induced by standard hormone cocktails to differentiate into mature adipocytes. Briefly, cells were induced with DMEM containing $10 \%$ FCS, $5 \mu \mathrm{g} / \mathrm{ml}$ insulin, $0.5 \mathrm{mM} \mathrm{IBMX}$, and $1 \mu \mathrm{M}$ dexamethasone for 2 days, treated with DMEM containing $5 \mu \mathrm{g} / \mathrm{ml}$ insulin for 4 days, and switched to DMEM containing 10\% FCS for 2 more days. On day 8 of differentiation, mature adipocytes were harvested for further experiments.

Isolation of stromal vascular fractions and differentiation into adipocytes. SVFs were isolated from iWATs of 6-8-week-old male WT, $C d 36^{-1-}$, or Rosa$\mathrm{Cr}{ }^{E R T 2} ; D h h c 5^{f / f}$ mice. SVFs were cultured and differentiated into mature adipocytes as 3T3-L1 cells.

Lentivirus production and infection. HEK293T cells were cultured in DMEM ( $4.5 \mathrm{~g} / \mathrm{L}$ glucose) supplemented with $10 \%(\mathrm{v} / \mathrm{v})$ FCS (Thermo Fisher Scientific), $100 \mathrm{U} / \mathrm{ml}$ penicillin, and $100 \mathrm{mg} / \mathrm{ml}$ streptomycin at $37^{\circ} \mathrm{C}$ in an atmosphere of $5 \%$ $\mathrm{CO}_{2}$. For lentivirus packaging, gene overexpressing or shRNA vector was cotransfected with psPAX2 and pMD2.G into HEK293T cells. Medium containing lentiviral particles were either concentrated at $70,000 \times g$ for $2 \mathrm{~h}$ or directly aliquoted and stored at $-80^{\circ} \mathrm{C}$ until use.
For lentiviral infections of 3T3-L1 adipocytes, 3T3-L1 cells, on day 4 of differentiation, were infected with lentivirus encoding scrambled shRNA or shRNA targeting a certain gene in medium containing $8-10 \mu \mathrm{g} / \mathrm{ml}$ polybrene. Cells were selected against $5 \mu \mathrm{g} / \mathrm{ml}$ puromycin on day 5 for at least $48 \mathrm{~h}$ before harvest for the described experiments.

Electroporation of adipocytes. On day 6 of differentiation, indicated adipocytes in the figure legends were trypsinized and resuspended in electroporation buffer (Lonza-Amaxa). Cells were aliquoted into cuvettes $\left(2 \times 10^{6}\right.$ cells $)$ and mixed with $2 \mu \mathrm{g}$ of indicated plasmid in the figure legends. Electroporation was performed using the A-033 program of Nucleofector ${ }^{\mathrm{TM}} 2 \mathrm{~b}$ (Lonza-Amaxa). After electroporation, cells were plated to $35-\mathrm{mm}$ dishes for further experiments.

Protein palmitoylation analysis by Acyl-RAC assay. Palmitoylated proteins were isolated using resin-assisted capture of S-acylated proteins (Acyl-RAC) ${ }^{56}$ with more stringent washing conditions ${ }^{20}$. Briefly, free thiol groups were blocked with $0.1 \% \mathrm{~s}$-methyl methanethiosulfate at $42{ }^{\circ} \mathrm{C}$ for $15 \mathrm{~min}$. Proteins were precipitated by cold acetone at $-20^{\circ} \mathrm{C}$ for $1 \mathrm{~h}$, washed twice by $70 \%$ acetone, and resuspended in $0.6 \mathrm{ml}$ Buffer A (100 mM Hepes, pH7.5, $1 \mathrm{mM}$ EDTA, $1 \%$ SDS). Samples were mixed with $20 \mu \mathrm{l}$ thiopropyl sepharose $6 \mathrm{~B}$, and incubated with $0.21 \mathrm{M} \mathrm{NH}_{2} \mathrm{OH}$ or $\mathrm{NaCl}$ with constant rotation at room temperature for $3 \mathrm{~h}$. Resins were washed with Buffer A containing $8 \mathrm{M}$ urea for five times $(5 \mathrm{~min}$ each) and eluted with $60 \mu \mathrm{l}$ Buffer A containing $50 \mathrm{mM}$ DTT at room temperature for $20 \mathrm{~min}$. Eluted fractions from Acyl-RAC assay were analyzed by sliver staining, mass spectrometry, or western blot. Blots were developed in a ChemStudio imaging system (Analytik Jena AG).

Immunoprecipitation and western blot. Fully differentiated 3T3-L1 adipocytes were lysed in Buffer B (50 mM Tris (pH7.4), $150 \mathrm{mM} \mathrm{NaCl}, 1 \%$ Triton, and $0.1 \%$ SDS) containing protease and phosphatase inhibitors (MCE). Cell lysate was immunoprecipitated with anti-DHHC5 (Sigma, HPA014670), anti-CD36 (Novus, NBP2-54790), anti-SYK (CST, 13198s), or anti-LYN (CST, 2796s) and pulled down with protein A/G beads. Pellet was washed five times with Buffer C $(50 \mathrm{mM}$ Tris (pH7.4), $150 \mathrm{mM} \mathrm{NaCl}$ and $0.1 \%$ Triton), and incubated with SDS loading buffer at $37^{\circ} \mathrm{C}$ for $1 \mathrm{~h}$

To generate pTyr91 antibody of DHHC5, a synthetic phospho-peptide CDKEDDFRAPL(pY)KTVE was conjugated to KLH and used to immunize rabbits. After three immunizations, anti-serum was affinity purified and used for western blot.

The following antibodies were used for western blot: anti-Flag (1:1000, SigmaAldrich, F1804), anti-HA (1:5000, Roche, 11867423001), anti-FABP4 (1:1000, Abclonal, A11481), anti-CD36 (1:1000, Sino Biological Inc., 80263-T48), antiCAV1 (1:1000, CST, 3238s), anti-DHHC5 (1:1000, Sigma-Aldrich, HPA014670), anti-pTyr 4G10 (1:1000, EMD Millipore, 05-321), anti-LYN (1:1000, CST, 2796s), anti-pLYN (Y396) (1:1000, Abcam, 226778), anti-JNK (1:1000, CST, 9252s), antipJNK (T183/Y185) (1:1000, CST, 9255s), anti-VAV2 (1:1000, Proteintech, 602111-Ig), anti-pVAV(Tyr174) (1:1000, Santa Cruz, SC-135788), anti-SYK (1:1000, CST, 13198s), anti-pSYK (Y525/526) (1:1000, CST, 2710s), and anti-GAPDH (1:1000, Proteintech, 60004-1-Ig).

Blots were developed and band intensities were quantified using VisionWorks software on a ChemStudio imaging system (Analytik Jena AG). Uncropped blots are available in the Source Data file.

Identification of interacting proteins of depalmitoylated CD36. On day 0 DHHC5 ${ }^{-1-}$ HEK293T cells were set up $7.5 \times 10^{5}$ cells per 10 -cm dish (five dishes). On day 2, cells were transfected with $6 \mu \mathrm{g}$ CD36-Flag/pCDH-puro. On day 3, cells were treated with 2 -bromopalmitate $(100 \mu \mathrm{M})$ for $24 \mathrm{~h}$. On day 4 , cells were 

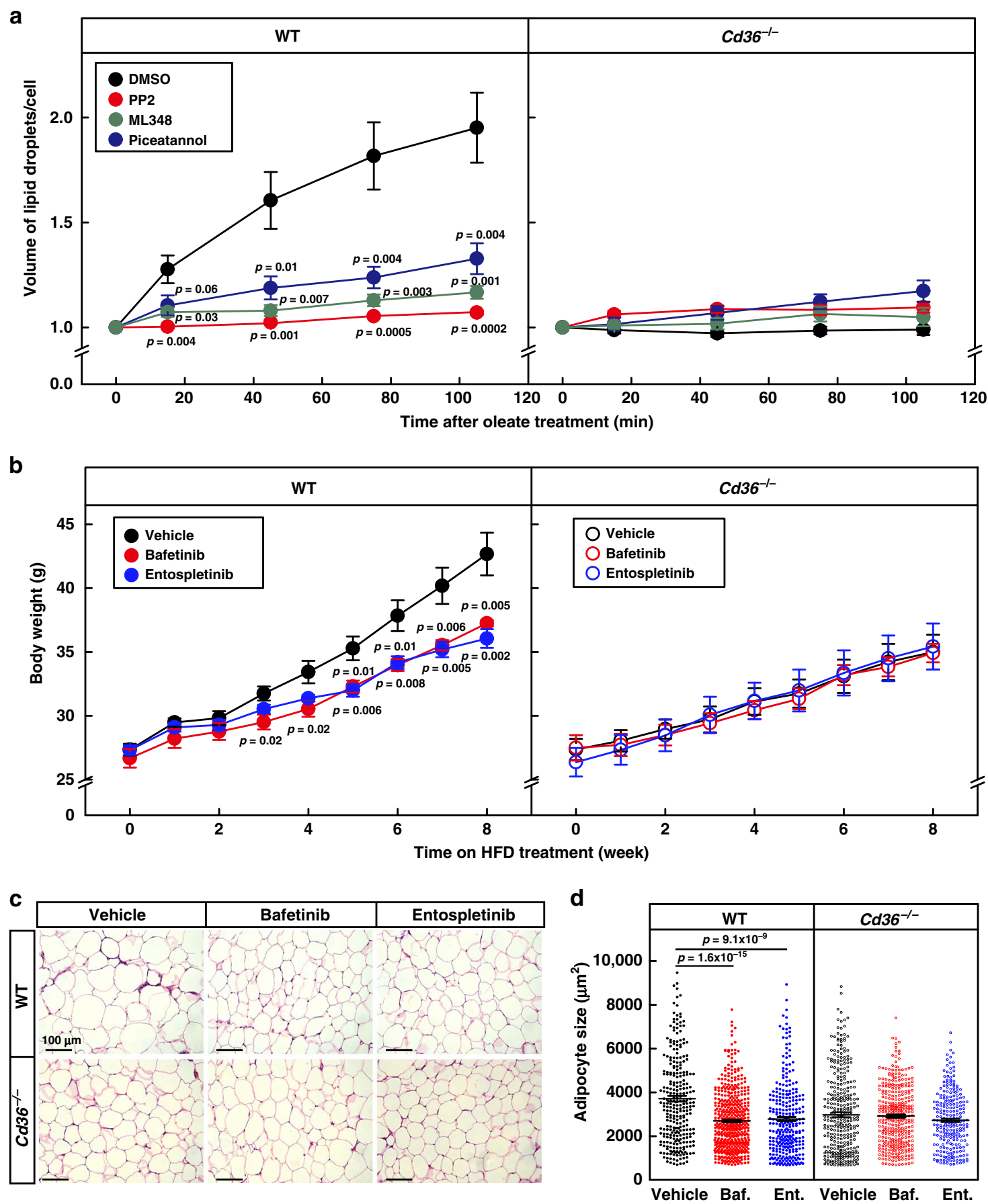

Fig. 7 Blocking endocytosis inhibits lipid droplet growth and HFD-induced obesity. a On the day of experiment, WT and Cd36-/- adipocytes were pretreated with PP2 $(20 \mu \mathrm{M}), \mathrm{ML3} 48(10 \mu \mathrm{M})$, or piceatannol $(40 \mu \mathrm{M})$ for $1 \mathrm{~h}$, then treated with oleate $(100 \mu \mathrm{M})$ and BODIPY $493 / 503(0.1 \mu \mathrm{g} / \mathrm{ml})$, and subjected to live imaging on a Zeiss LSM-780 confocal microscope in a 3D Z-stack mode for $2 \mathrm{~h}$. The number of cells was $12,9,8$, and 10 for DMSO, PP2, ML348, and piceatannol-treated WT cells, and 8, 8, 8, and 10 for corresponding groups in Cd36 $6^{-/-}$cells, respectively. Total volume of lipid droplets in each cell was calculated. Each value represents mean \pm SEM, and the volume at 0 min was normalized to 1.0. Two-sided Student's $t$ test was performed between DMSO- and PP2-, ML348- or piceatannol-treated cells. b-d WT and Cd36-/- mice (8-week-old male, $n=6 /$ group) were daily gavaged with vehicle $(0.5 \%$ methyl cellulose), bafetinib $(20 \mathrm{mg} / \mathrm{kg})$, or entospletinib $(10 \mathrm{mg} / \mathrm{kg})$ at 7 pm and subjected to HFD feeding for 8 weeks. b Body weight of the mice was monitored every week. c, d Gonadal WAT was subjected to H\&E staining, and representative pictures were shown (c). Scale bar, $100 \mu \mathrm{m}$. Quantification of the surface area of adipocytes in gWAT by ImageJ. The size of each adipocyte was quantified by ImageJ and plotted as mean \pm SEM from 287, 488, 305, 324, 362, and 218 cells, respectively d. Two-sided Student's $t$ test was performed between vehicle and bafetinib (Baf.), or entospletinib (Ent.) treated group, respectively. These experiments were repeated twice. Source data are provided as a Source Data file. 


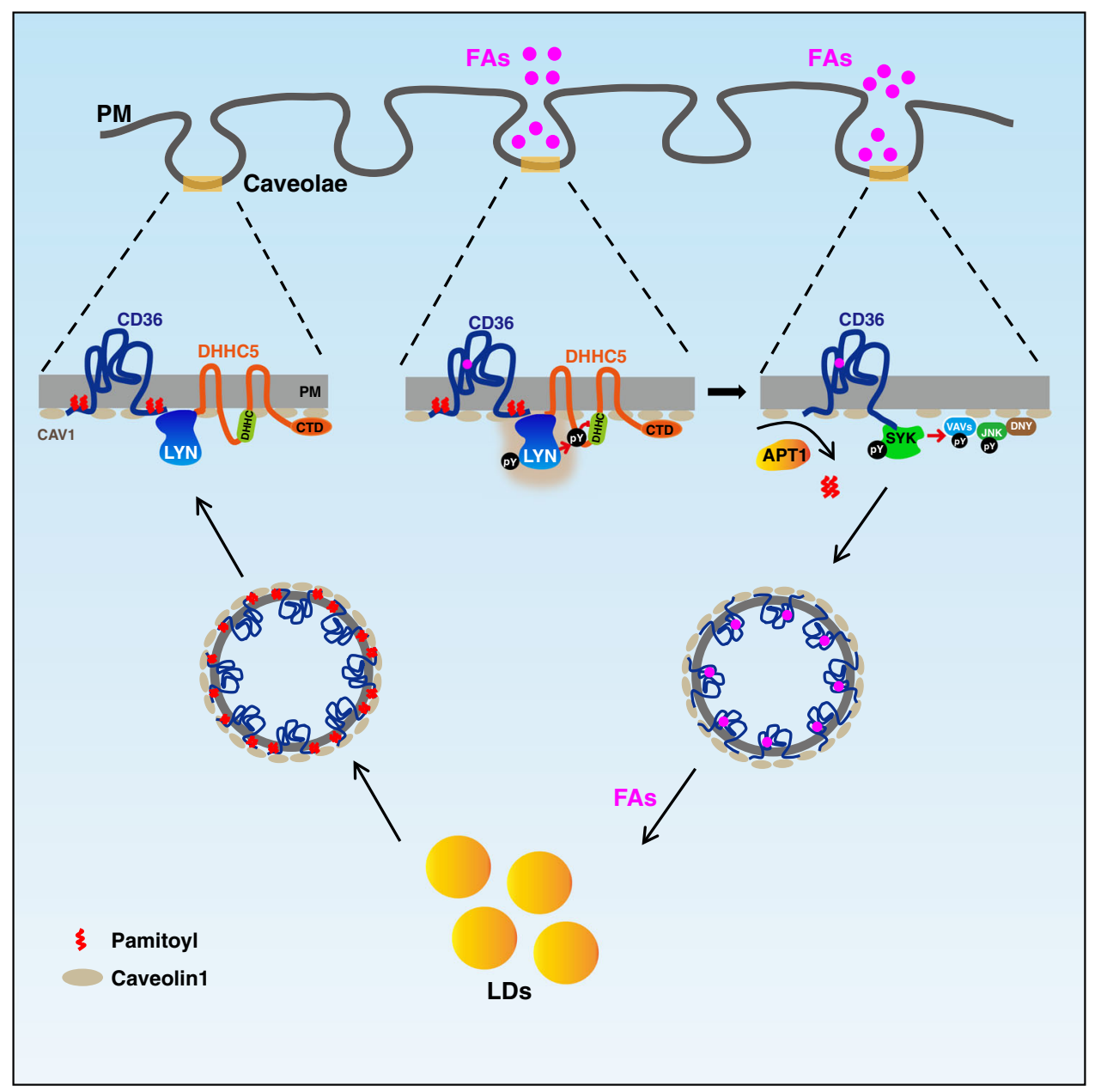

Fig. 8 A working model of FA uptake by CD36-mediated caveolar endocytosis. The plasma membrane of adipocytes is enriched with caveolaes. In a caveolae, CD36 is in the palmitoylated form and its FA binding cavity is on the outer layer, whereas CAV1 is on the inner layer. When FAs bind to CD36, a SRC kinase LYN gets activated, and it phosphorylates DHHC5 at Tyr91 and inactivates DHHC5, resulting in the subsequent depalmitoylation of CD36 by the depalmitoylase APT1. The depalmitoylated CD36 then recruits SYK to phosphorylate VAV and JNK, thereby initiating CD36-mediated caveolar endocytosis. The endocytosed vesicles deliver FAs to lipid droplets for storage. After that, CD36 gets re-palmitoylated and recycled to the plasma membrane for another round of delivery.

harvested and immunoprecipitated with anti-Flag M2 beads. Meanwhile, about $3 \mathrm{~g}$ gWAT were collected from mice that were fasted and refed with HFD for $4 \mathrm{~h}$ Tissues were then homogenized in Buffer B, and the resultant supernatant was incubated with the M2 beads at $4{ }^{\circ} \mathrm{C}$ for $4 \mathrm{~h}$. The beads were eluted with $3 \times$ Flag peptides $(0.2 \mathrm{mg} / \mathrm{ml})$, and the eluted proteins were separated on SDS-PAGE, and subjected to silver staining and mass spectrometry analysis.

Surface biotinylation. Briefly, cells were incubated with freshly prepared sulfoNHS-SS-biotin $(0.5 \mathrm{mg} / \mathrm{ml})$ in PBS for $30 \mathrm{~min}$ on ice. Cells were then washed three times with ice-cold PBS containing $50 \mathrm{mM}$ glycine. Cells were lysed in PBS containing $1 \%$ triton $\mathrm{X}-100$, and supernatant was incubated with streptavidin agarose at $4{ }^{\circ} \mathrm{C}$ for $2 \mathrm{~h}$. Beads were washed five times with PBS containing $1 \%$ triton X-100, eluted with SDS loading buffer at $37^{\circ} \mathrm{C}$ for $1 \mathrm{~h}$, and subjected to western blot analysis.

Immunofluorescence. After indicated treatments in the figure legends, cells were fixed with $4 \%$ paraformaldehyde for $15 \mathrm{~min}$ at room temperature, permeabilized with $0.05 \%$ NP40 and $0.05 \%$ saponin in PBS for 5 min, and blocked with 5\% BSA in PBS for $1 \mathrm{~h}$. Cells were then incubated with anti-CD36 (1:100, Abcam, ab23680), anti-CAV1 (1:100, CST, 3238s), anti-Flag antibody (1:100, Sigma-Aldrich, F1804), or anti-HA (1:100, Roche, 11867423001$)$ overnight at $4{ }^{\circ} \mathrm{C}$ followed by three times wash with PBS, and then incubated with Alexa Fluor 488 goat anti-rabbit IgG secondary antibody (1:200, Thermo Fisher Scientific) or Alexa Fluor 594 goat antimouse IgG secondary antibody (1:200, Thermo Fisher Scientific) for $3 \mathrm{~h}$ at $4{ }^{\circ} \mathrm{C}$ followed by another three washed with PBS. Cells were then stained with LipidTOX (Thermo Fisher, H34477) and washed three times with PBS. Cover slides were then mounted. Images were taken using a Zeiss LSM-780 confocal microscopy. Imaris 9.2.0 was used for $3 \mathrm{D}$ reconstruction.
PacFA labeling of 3T3-L1 adipocytes. PacFA labeling was modified from a previous study ${ }^{23}$. 3T3-L1 adipocytes were pretreated with serum-free medium for $4 \mathrm{~h}$, and then treated with BSA or BSA-conjugated PacFA $(50 \mu \mathrm{M})$ for $20 \mathrm{~min}$. Cells were washed three times with PBS containing $1 \%$ BSA and subjected to UV crosslinking on ice for $30 \mathrm{~min}$.

For fluorescent detection of PacFA, cells were fixed with methanol at $-20^{\circ} \mathrm{C}$ for $20 \mathrm{~min}$ and then subjected to click chemistry in $1 \mathrm{mM}$ ascorbic acid, $0.1 \mathrm{mM}$ TBTA, $1 \mathrm{mM} \mathrm{CuSO} 4$, and $2 \mu \mathrm{M}$ Alexa 488 azide (Thermo Fisher, A10266) at $25^{\circ} \mathrm{C}$ for $1 \mathrm{~h}$. Cells were then washed with PBS and subjected to immunostaining of CD36 as above.

For isolation of PacFA-labeled proteins, cells were lysed in PBS containing 1\% SDS supplemented with protease inhibitors and subjected to click chemistry in $1 \mathrm{mM}$ ascorbic acid, $0.1 \mathrm{mM}$ TBTA, $1 \mathrm{mM} \mathrm{CuSO}_{4}$, and $1 \mathrm{mM}$ biotin azide (Thermo Fisher, B10184) at $25^{\circ} \mathrm{C}$ for $1 \mathrm{~h}$. To remove free biotin azide, cell lysate was precipitated with cold acetone (three volumes) at $-20^{\circ} \mathrm{C}$ for $1 \mathrm{~h}$, and the resultant pellet was washed twice with cold $70 \%$ acetone. Pellet was dried, resuspended in PBS containing $0.1 \%$ SDS, and incubated with streptavidin beads at $4{ }^{\circ} \mathrm{C}$ for $1 \mathrm{~h}$. Beads were washed three times with PBS containing $0.1 \%$ SDS and then eluted with Buffer D (50 mM Tris, pH7.4, $150 \mathrm{mM} \mathrm{NaCl}$, 2\% SDS, 0.1 M Urea, 5 mM EGTA, $5 \mathrm{mM}$ EDTA, $5 \mathrm{mM}$ biotin, and $10 \%$ DMSO). The eluted fraction was then subjected to immunoblotting with indicated antibodies.

Fatty acid uptake. For FA uptake in preadipocytes, cells were pretreated in serumfree medium for $4 \mathrm{~h}$, and PP2 $(20 \mu \mathrm{M})$, ML348 $(10 \mu \mathrm{M})$, or piceatannol $(40 \mu \mathrm{M})$ for $1 \mathrm{~h}$, followed by treatment with $100 \mu \mathrm{M}$ BSA-conjugated oleate for $4 \mathrm{~h}$. Cells were then stained with BODIPY 493/503 (Thermo Fisher, D3922). Images were taken under a Zeiss LSM-780 confocal microscopy using an excitation wavelength of $488 \mathrm{~nm}$ with the same laser intensity. None of the images were overexposed. 
Fluorescent intensity of BODIPY was quantified from 20 cells of each treatment using ZEN 2010.

For ${ }^{3} \mathrm{H}$-oleate uptake in adipocytes, cells were pretreated with the same inhibitors as above, and then treated with $100 \mu \mathrm{M}$ BSA-conjugated ${ }^{3} \mathrm{H}$-oleate (specific activity, $2268 \mathrm{dpm} / \mathrm{nmol}$ ) for $1 \mathrm{~h}$. Cells were washed twice with cold PBS containing $0.2 \%$ BSA twice, once with cold PBS, and extracted with hexane: isopropanol (3:2). The extracted fractions were subjected to scintillation counting. After extraction, the cells on the dish were dried out for measurement of protein content by a Pierce BCA protein assay kit.

Growth of lipid droplets. Adipocytes were pretreated with serum-free medium for $4 \mathrm{~h}$, and indicated inhibitors for $1 \mathrm{~h}$ in the figure legends. Cells were then treated with BSA-conjugated oleate $(100 \mu \mathrm{M})$ and BODIPY 493/503 $(0.1 \mu \mathrm{g} / \mathrm{ml})$, and subjected to live imaging on a Zeiss LSM-780 confocal microscope in a 3D Z-stack mode for $2 \mathrm{~h}$. In all, 8-12 cells of each treatment were imaged. Images were reconstructed with Imaris 9.2.0, and volume of lipid droplets per cell was calculated.

Bafetinib and entospletinib treatment and HFD feeding. WT and $C d 36^{-1-}$ mice (8-week-old male, $n=6 /$ group) were daily gavaged with vehicle $(0.5 \%$ methyl cellulose), bafetinib $(20 \mathrm{mg} / \mathrm{kg})$, or entospletinib $(10 \mathrm{mg} / \mathrm{kg})$ at $7 \mathrm{p} . \mathrm{m}$. and subjected to HFD (Research Diet, D12492) feeding for 8 weeks. Glucose tolerance test was performed on week 8 as previously described ${ }^{20}$. At the end of the experiment, mice were dissected and H\&E staining of gWAT was performed as previously described $^{20}$.

Quantitative real-time PCR. Total RNA was isolated using Trizol, and quantitative real-time PCR was performed according to Zhao et al. ${ }^{57}$. Briefly, RNA was reversed transcribed using a kit form Toyobo (FSQ-301), and real-time PCR was performed using a SYBR green master mix (Yeasen, 11203ES08). The primers are listed in Supplementary Table 1 . The mRNA of 36B4 was used as the invariant control.

Electron microscopy. 3T3-L1 adipocytes were cultured and differentiated as above. After trypsinization, cells were fixed in $2.5 \%$ glutaraldehyde for $2-3 \mathrm{~h}$ at $4{ }^{\circ} \mathrm{C}$. After fixation, three volumes of $0.1 \mathrm{M}$ PBS were added to the fixation buffer, and cells were washed three times with $0.1 \mathrm{M}$ PBS. Samples were then postfixed with $1 \% \mathrm{OsO} 4$, embedded, and sectioned. Specimens were visualized on a Hitachi HT-7800 transmission election microscope.

Quantification and statistical analysis. All the statistical analysis was performed by Student's two-tailed paired $t$ test using EXCEL2010. All the values represent mean \pm SEM. All the statistical details of the experiments can be found in the figure legends, including exact number of cells or mice. No data were excluded from any of the experiments.

\section{Data availability}

All data generated or analyzed during this study are included in this published article and its supplementary information files. Source data are provided with this paper.

Received: 28 January 2020; Accepted: 31 August 2020;

Published online: 21 September 2020

\section{References}

1. McArthur, M. J. et al. Cellular uptake and intracellular trafficking of long chain fatty acids. J. Lipid Res. 40, 1371-1383 (1999).

2. Hamilton, J. A. Fatty acid transport: difficult or easy? J. Lipid Res. 39, 467-481 (1998).

3. Hamilton, J. A. Fast flip-flop of cholesterol and fatty acids in membranes: implications for membrane transport proteins. Curr. Opin. Lipidol. 14, 263-271 (2003).

4. Hajri, T. \& Abumrad, N. A. Fatty acid transport across membranes: relevance to nutrition and metabolic pathology. Annu. Rev. Nutr. 22, 383-415 (2002)

5. Ehehalt, R. et al. Translocation of long chain fatty acids across the plasma membrane-lipid rafts and fatty acid transport proteins. Mol. Cell. Biochem. 284, 135-140 (2006).

6. Pepino, M. Y., Kuda, O., Samovski, D. \& Abumrad, N. A. Structure-function of CD36 and importance of fatty acid signal transduction in fat metabolism. Annu. Rev. Nutr. 34, 281-303 (2014).

7. Su, X. \& Abumrad, N. A. Cellular fatty acid uptake: a pathway under construction. Trends Endocrinol. Metab. 20, $72-77$ (2009).

8. Pohl, J., Ring, A., Korkmaz, U., Ehehalt, R. \& Stremmel, W. FAT/CD36mediated long-chain fatty acid uptake in adipocytes requires plasma membrane rafts. Mol. Biol. Cell 16, 24-31 (2005).
9. Lisanti, M. P. et al. Characterization of caveolin-rich membrane domains isolated from an endothelial-rich source: implications for human disease. J. Cell Biol. 126, 111-126 (1994).

10. Ring, A., Le Lay, S., Pohl, J., Verkade, P. \& Stremmel, W. Caveolin-1 is required for fatty acid translocase (FAT/CD36) localization and function at the plasma membrane of mouse embryonic fibroblasts. Biochim. Biophys. Acta 1761, 416-423 (2006)

11. Coburn, C. T. et al. Defective uptake and utilization of long chain fatty acids in muscle and adipose tissues of CD36 knockout mice. J. Biol. Chem. 275, 32523-32529 (2000)

12. Tanaka, T. et al. Defect in human myocardial long-chain fatty acid uptake is caused by FAT/CD36 mutations. J. Lipid Res. 42, 751-759 (2001).

13. Hames, K. C., Vella, A., Kemp, B. J. \& Jensen, M. D. Free fatty acid uptake in humans with CD36 deficiency. Diabetes 63, 3606-3614 (2014).

14. Abumrad, N. A., El-Maghrabi, M. R., Amri, E. Z., Lopez, E. \& PA, G. Cloning of a rat adipocyte membrane protein implicated in binding or transport of long-chain fatty acids that is induced during preadipocyte differentiation. J. Biol. Chem. 268, 17665-17668 (1993).

15. Xu, S., Jay, A., Brunaldi, K., Huang, N. \& Hamilton, J. A. CD36 enhances fatty acid uptake by increasing the rate of intracellular esterification but not transport across the plasma membrane. Biochemistry 52, 7254-7261 (2013).

16. Resh, M. D. Trafficking and signaling by fatty-acylated and prenylated proteins. Nat. Chem. Biol. 2, 584-590 (2006).

17. Linder, M. E. \& Deschenes, R. J. Palmitoylation: policing protein stability and traffic. Nat. Rev. Mol. Cell Biol. 8, 74-84 (2007).

18. Fukata, Y., Murakami, T., Yokoi, N. \& Fukata, M. Local palmitoylation cycles and specialized membrane domain organization. Curr. Top. Membr. 77, 97-141 (2016).

19. Peng, T., Thinon, E. \& Hang, H. C. Proteomic analysis of fatty-acylated proteins. Curr. Opin. Chem. Biol. 30, 77-86 (2016).

20. Wang, J. et al. DHHC4 and DHHC5 facilitate fatty acid uptake by palmitoylating and targeting CD36 to the plasma membrane. Cell Rep. 26, 209-221.e205 (2019).

21. Doherty, G. J. \& McMahon, H. T. Mechanisms of endocytosis. Annu. Rev. Biochem. 78, 857-902 (2009).

22. Kuda, O. et al. Sulfo-N-succinimidyl oleate (SSO) inhibits fatty acid uptake and signaling for intracellular calcium via binding CD36 lysine 164: SSO also inhibits oxidized low density lipoprotein uptake by macrophages. J. Biol. Chem. 288, 15547-15555 (2013).

23. Haberkant, P. et al. In vivo profiling and visualization of cellular protein-lipid interactions using bifunctional fatty acids. Angew. Chem. 52, 4033-4038 (2013).

24. Lin, D. T. \& Conibear, E. ABHD17 proteins are novel protein depalmitoylases that regulate N-Ras palmitate turnover and subcellular localization. Elife 4, e11306 (2015).

25. Dekker, F. J. et al. Small-molecule inhibition of APT1 affects Ras localization and signaling. Nat. Chem. Biol. 6, 449-456 (2010).

26. Won, S. J. et al. Molecular mechanism for isoform-selective inhibition of Acyl protein thioesterases 1 and 2 (APT1 and APT2). ACS Chem. Biol. 11, 3374-3382 (2016)

27. Rana, M. S. et al. Fatty acyl recognition and transfer by an integral membrane S-acyltransferase. Science 359, eaao6326 (2018).

28. Li, Y., Martin, B. R., Cravatt, B. F. \& Hofmann, S. L. DHHC5 protein palmitoylates flotillin-2 and is rapidly degraded on induction of neuronal differentiation in cultured cells. J. Biol. Chem. 287, 523-530 (2012).

29. Hanke, J. H. et al. Discovery of a novel, potent, and Src family-selective tyrosine kinase inhibitor. Study of Lck- and FynT-dependent T cell activation. J. Biol. Chem. 271, 695-701 (1996).

30. Nabi, I. R. \& Le, P. U. Caveolae/raft-dependent endocytosis. J. Cell Biol. 161, 673-677 (2003).

31. Rahaman, S. O. et al. A CD36-dependent signaling cascade is necessary for macrophage foam cell formation. Cell Metab. 4, 211-221 (2006).

32. Rahaman, S. O., Swat, W., Febbraio, M. \& Silverstein, R. L. Vav family Rho guanine nucleotide exchange factors regulate $\mathrm{CD} 36$-mediated macrophage foam cell formation. J. Biol. Chem. 286, 7010-7017 (2011).

33. Rahaman, S. O., Zhou, G. \& Silverstein, R. L. Vav protein guanine nucleotide exchange factor regulates $\mathrm{CD} 36$ protein-mediated macrophage foam cell formation via calcium and dynamin-dependent processes. J. Biol. Chem. 286, 36011-36019 (2011)

34. Wilkinson, B., Koenigsknecht-Talboo, J., Grommes, C., Lee, C. Y. \& Landreth, G. Fibrillar beta-amyloid-stimulated intracellular signaling cascades require Vav for induction of respiratory burst and phagocytosis in monocytes and microglia. J. Biol. Chem. 281, 20842-20850 (2006)

35. Berton, G., Mocsai, A. \& Lowell, C. A. Src and Syk kinases: key regulators of phagocytic cell activation. Trends Immunol. 26, 208-214 (2005).

36. Heit, B. et al. Multimolecular signaling complexes enable Syk-mediated signaling of CD36 internalization. Dev. Cell 24, 372-383 (2013). 
37. Kimura, S. et al. NS-187, a potent and selective dual Bcr-Abl/Lyn tyrosine kinase inhibitor, is a novel agent for imatinib-resistant leukemia. Blood 106, 3948-3954 (2005).

38. Currie, K. S. et al. Discovery of GS-9973, a selective and orally efficacious inhibitor of spleen tyrosine kinase. J. Med. Chem. 57, 3856-3873 (2014).

39. Thorn, H. et al. Cell surface orifices of caveolae and localization of caveolin to the necks of caveolae in adipocytes. Mol. Biol. Cell 14, 3967-3976 (2003)

40. Parton, R. G. \& Simons, K. The multiple faces of caveolae. Nat. Rev. Mol. Cell Biol. 8, 185-194 (2007)

41. Wilfling, F. et al. Triacylglycerol synthesis enzymes mediate lipid droplet growth by relocalizing from the ER to lipid droplets. Dev. Cell 24, 384-399 (2013).

42. Jacquier, N. et al. Lipid droplets are functionally connected to the endoplasmic reticulum in Saccharomyces cerevisiae. J. Cell Sci. 124, 2424-2437 (2011).

43. $\mathrm{Xu}, \mathrm{N}$. et al. The FATP1-DGAT2 complex facilitates lipid droplet expansion at the ER-lipid droplet interface. J. Cell Biol. 198, 895-911 (2012).

44. Goldstein, J. L. \& Brown, M. S. The LDL receptor. Arterioscler. Thromb. Vasc. Biol. 29, 431-438 (2009).

45. Silverstein, R. L. \& Febbraio, M. CD36, a scavenger receptor involved in immunity, metabolism, angiogenesis, and behavior. Sci. Signal. 2, re3 (2009).

46. Ge, L. et al. The cholesterol absorption inhibitor ezetimibe acts by blocking the sterol-induced internalization of NPC1L1. Cell Metab. 7, 508-519 (2008).

47. Zhang, Y. Y. et al. A LIMA1 variant promotes low plasma LDL cholesterol and decreases intestinal cholesterol absorption. Science 360, 1087-1092 (2018).

48. Greaves, J. \& Chamberlain, L. H. DHHC palmitoyl transferases: substrate interactions and (patho)physiology. Trends Biochem. Sci. 36, 245-253 (2011).

49. Parsons, S. J. \& Parsons, J. T. Src family kinases, key regulators of signal transduction. Oncogene 23, 7906-7909 (2004).

50. Le Lay, S. et al. Cholesterol-induced caveolin targeting to lipid droplets in adipocytes: a role for caveolar endocytosis. Traffic 7, 549-561 (2006).

51. Pol, A. et al. Cholesterol and fatty acids regulate dynamic caveolin trafficking through the Golgi complex and between the cell surface and lipid bodies. Mol. Biol. Cell 16, 2091-2105 (2005).

52. Zeke, A., Misheva, M., Remenyi, A. \& Bogoyevitch, M. A. JNK signaling: regulation and functions based on complex protein-protein partnerships. Microbiol. Mol. Biol. Rev. 80, 793-835 (2016).

53. Febbraio, M. et al. A null mutation in murine CD36 reveals an important role in fatty acid and lipoprotein metabolism. J. Biol. Chem. 274, 19055-19062 (1999).

54. Hannah, V. C., Ou, J., Luong, A., Goldstein, J. L. \& Brown, M. S. Unsaturated fatty acids down-regulate srebp isoforms 1a and 1c by two mechanisms in HEK-293 cells. J. Biol. Chem. 276, 4365-4372 (2001).

55. Moffat, J. et al. A lentiviral RNAi library for human and mouse genes applied to an arrayed viral high-content screen. Cell 124, 1283-1298 (2006).

56. Forrester, M. T. et al. Site-specific analysis of protein S-acylation by resinassisted capture. J. Lipid Res. 52, 393-398 (2011).

57. Zhao, T. J. et al. Ghrelin secretion stimulated by $\beta 1$-adrenergic receptors in cultured ghrelinoma cells and in fasted mice. Proc. Natl Acad. Sci. USA 107, 15868-15873 (2010).

\section{Acknowledgements}

We thank Drs. Peng Li and Bao-Liang Song for critical reading of the paper. This work was supported by the National Key R\&D Program of China (2016YFA0502003 to T.-J.Z.), the National Natural Science Foundation of China (91857107, 31671223, and 31871193 to T.-J.Z; 81772818 to H.G), the Fundamental Research Funds for the Central Universities (20720180046 to H.G.), Project "111" sponsored by the State Bureau of Foreign Experts and Ministry of Education of China (BP2018017)

\section{Author contributions}

J.-W.H., J.W., H.G., H.-H.S., Y.-Y.Z., Y.-F.L., X.-Y.L., N.Z., and X.W. performed the experiments. C.X., L.H., X.H., H.-R.W., C.-B.L., and B.L. provided expertise and materials. S.C. and T.-J.Z. designed the experiments, analyzed the data, and wrote the paper.

\section{Competing interests}

The authors declare no competing interests.

\section{Additional information}

Supplementary information is available for this paper at https://doi.org/10.1038/s41467 020-18565-8.

Correspondence and requests for materials should be addressed to S.C. or T.-J.Z.

Peer review information Nature Communications thanks Nada Abumrad and other, anonymous, reviewers for their contributions to the peer review of this work. Peer review reports are available.

Reprints and permission information is available at http://www.nature.com/reprints

Publisher's note Springer Nature remains neutral with regard to jurisdictional claims in published maps and institutional affiliations.

Open Access This article is licensed under a Creative Commons Attribution 4.0 International License, which permits use, sharing, adaptation, distribution and reproduction in any medium or format, as long as you give appropriate credit to the original author(s) and the source, provide a link to the Creative Commons license, and indicate if changes were made. The images or other third party material in this article are included in the article's Creative Commons license, unless indicated otherwise in a credit line to the material. If material is not included in the article's Creative Commons license and your intended use is not permitted by statutory regulation or exceeds the permitted use, you will need to obtain permission directly from the copyright holder. To view a copy of this license, visit http://creativecommons.org/ licenses/by/4.0/

(C) The Author(s) 2020 